\title{
Insight into the roles of melatonin in bone tissue and bone-related diseases (Review)
}

\author{
XIAOFENG LU ${ }^{1-3^{*}}$, SHAOLING YU ${ }^{1-3^{*}}$, GUANGJIN CHEN ${ }^{1-3}$, WENHAO ZHENG $^{1-3}$, \\ JINFENG PENG ${ }^{1-3}$, XIAOFEI HUANG ${ }^{1-3}$ and LILI CHEN ${ }^{1-3}$
}

\author{
${ }^{1}$ Department of Stomatology, Union Hospital, Tongji Medical College, Huazhong University of Science and Technology, \\ Wuhan, Hubei 430022; ${ }^{2}$ School of Stomatology, Tongji Medical College, Huazhong University of Science and Technology, \\ Wuhan, Hubei 430030; ${ }^{3}$ Hubei Province Key Laboratory of Oral and Maxillofacial Development \\ and Regeneration, Wuhan, Hubei 430022, P.R. China
}

Received August 26, 2020; Accepted February 17, 2021

DOI: $10.3892 /$ ijmm.2021.4915

\begin{abstract}
Bone-related diseases comprise a large group of common diseases, including fractures, osteoporosis and osteoarthritis (OA), which affect a large number of individuals, particularly the elderly. The progressive destruction and loss of alveolar bone caused by periodontitis is a specific type of bone loss, which has a high incidence and markedly reduces the quality of life of patients. With the existing methods of prevention and treatment, the incidence and mortality of bone-related diseases are still gradually increasing, creating a significant financial burden to societies worldwide. To prevent the occurrence of bone-related diseases, delay their progression or reverse the injuries they cause, new alternative or complementary treatments need to be developed. Melatonin exerts numerous physiological effects, including inducing anti-inflammatory and antioxidative functions, resetting circadian rhythms and promoting wound healing and tissue regeneration. Melatonin
\end{abstract}

Correspondence to: Professor Lili Chen, Department of Stomatology, Union Hospital, Tongji Medical College, Huazhong University of Science and Technology, 1277 Jiefang Avenue, Wuhan, Hubei 430022, P.R. China

E-mail: chenlili1030@hust.edu.cn

${ }^{*}$ Contributed equally

Abbreviations: ALP, alkaline phosphatase; CAL, clinical attachment level; DM, diabetes mellitus; ECM, extracellular matrix; GC, glucocorticoid; i.p. injection, intraperitoneal injection; L/D, light/dark; MSCs, mesenchymal stem cells; MTNR1A, melatonin receptor 1A; NSPT, nonsurgical periodontal therapy; OA, osteoarthritis; OCN, osteocalcin; OPG, osteoprotegerin; PD, probing depth; RANKL, receptor activator of NF- $\mathrm{BB}$ ligand; ROS, reactive oxygen species, SRP, scaling and root planning; TGF, transforming growth factor; TNF, tumor necrosis factor; VEGF, vascular endothelial growth factor

Key words: melatonin, bone injury, osteoporosis, osteoarthritis, periodontitis also participates in the health management of bone and cartilage. In the present review, the potential roles of melatonin in the pathogenesis and progression of bone injury, osteoporosis, OA and periodontitis are summarized. Furthermore, the high efficiency and diversity of the physiological regulatory effects of melatonin are highlighted and the potential benefits of the use of melatonin for the clinical prevention and treatment of bone-related diseases are discussed.

\section{Contents}

1. Introduction

2. Bone injury

3. Osteoporosis

4. Osteoarthritis

5. Periodontitis

6. Differential effects of melatonin administered at various concentrations and times

7. Conclusion and future prospects

\section{Introduction}

Bone-related diseases, such as fractures, osteoporosis and osteoarthritis (OA), severely affect the quality of life of patients due to their high incidence, slow recovery, associated pain and negative effects on patient behavior. Due to the aging of the population in several countries, the prevalence of bone-related diseases is projected to further increase in the following few decades. Data from 2013 indicated that $~ 57$ million Americans aged $>50$ years suffered from bone diseases, among which 48 million had osteopenia and 9 million had osteoporosis. The diseases were accompanied by a risk of fracture in these individuals. Without intervention, the prevalence of osteopenia is projected to increase to 64.3 million American individuals and that of osteoporosis to 11.9 million by the year 2030 (1). Bone disease is the focus of a large number of clinical studies, due to the accompanying high combined lifetime risk of forearm, hip and vertebral fractures (40\%), which is comparable to the rate of cardiovascular diseases (2). In addition to the high morbidity 
and mortality rates, fractures related to osteoporosis have also created a significant financial burden to societies worldwide. For instance, osteoporosis-related costs in the European Union amounted to $€ 37$ billion in 2010 ; the majority of that amount was spent on fracture therapy and long-term fracture care, accounting for 66 and 29\%, respectively, of the budget (3).

Melatonin, a common molecule with a simple structure known as $\mathrm{N}$-acetyl-5-methoxytryptamine, exists in almost all living organisms (4). Melatonin is secreted by pinealocytes in the pineal gland, while certain tissues can also produce a small amount of melatonin locally (5). There is a synchronization between melatonin production and the light/dark (L/D) cycle. Melatonin is synthesized and secreted in dark environments. When retinal photoreceptive ganglion cells are stimulated by light (mainly in the blue range), the synthesis and secretion of melatonin reduces until no more is secreted $(6,7)$. Generally, levels of melatonin begin to increase early in the evening and peak at 12-2 a.m., followed by a progressive decrease thereafter (8). As the organism ages, melatonin production gradually declines. Melatonin levels continuously decrease from the age of 40-45 years (6).

Currently, melatonin is considered a potent cytoprotective agent, rather than a hormone in the classical sense (9). Melatonin has excellent lipophilic properties and can easily enter the cell membrane and subcellular compartment (10). Melatonin can synchronize the circadian clock in peripheral tissues, maintain the synchronization of bone metabolism with L/D cycles and participate in numerous important physiological processes, such as anti-inflammatory, antitumor and antioxidation processes, as well as regulating circadian and endocrine rhythms, regulating immunity, and promoting wound healing and tissue regeneration $(7,11)$. Melatonin plays a positive role in bone-related diseases by exerting multiple effects. Although there are several physical and drug treatments for bone-related diseases, melatonin has the advantage over other drugs of being inexpensive, and having a wide safety margin, a wide impact on tissues and almost no side effect, suggesting its potential as a main or complementary treatment strategy for a large range of bone diseases.

\section{Bone injury}

Basic study of bone injury. Bone injury is very common in clinical practice. A variety of pathologies, such as tumors, trauma and surgery, as well as other factors, are likely to cause varying degrees of bone injury. This is a major issue for clinical treatment at present, and an important challenge that will threaten human health in the ensuing 50 years. The bone defect size largely determines the amount of bone repair. The larger the defect, the more difficult to obtain a satisfactory repair (12). Bone repair is often affected by a variety of negative effects, such as possible infection and ischemia of the bone injury site or adjacent tissues, and systemic diseases. Therefore, clinical intervention is required for bone repair.

Possible effects of melatonin on bone injury repair. As shown in Fig. 1, considerable evidence has demonstrated that melatonin contributes to bone repair. There are different claims about the effects of melatonin on osteogenic and osteoclastic activities. Melatonin has been shown to enhance the vertical bone augmentation of rat calvaria by increasing new bone regeneration, neovascularization and the number of osteoblast-like cells (13). It has also been shown to increase the cartilage and callus at the fracture site (14). At pharmacological doses, melatonin has been shown to stimulate osteoblast proliferation and alkaline phosphatase (ALP) activity in a dose-dependent manner. In vitro, melatonin increases the expression of collagen type I (Col1), bone sialoprotein, osteopontin and osteocalcin (OCN), and promotes the production of mineralized extracellular matrix (ECM). At the same time, femoral neocortical bone is enhanced in mice administered an intraperitoneal (i.p.) injection of melatonin (15). However, Histing et al (14) reported that melatonin delayed bone healing in a mouse model of femoral fracture. They explained that melatonin exerted a positive effect on bone repair by inhibiting bone resorption instead of promoting bone regeneration. The levels of receptor activator of $\mathrm{NF}-\kappa \mathrm{B}$ ligand (RANKL) were reduced in the mice with fractures that received a daily dose of $50 \mathrm{mg} / \mathrm{kg}$ melatonin, which may inhibit bone resorption by damaging the balance between osteoprotegerin (OPG) and RANKL.

In addition to exhibiting osteogenic and osteoclast activity, the role of osteoblast and osteoclast differentiation during the process of bone repair is also of interest. Sethi et al (16) noted that osteoblast differentiation requires chronic and uninterrupted melatonin exposure. Melatonin may promote mesenchymal stem cell (MSC) proliferation and migration, at least partly by upregulating neuropeptide Y (NPY) and NPY receptor Y1 (NPY1R), accelerating osteogenic differentiation and promoting fracture healing in the rat femur. Concurrently, NPY/NPY1R expression has been shown to be increased in the fracture zone and serum (17). Osteogenic and chondrogenic differentiation can be promoted, and adipogenesis can be inhibited through the enhancement of runt-related transcription factor 2 expression and the $\mathrm{Wnt} / \beta$-catenin signaling pathway, as well as the inhibition of peroxisome proliferator-activated receptor (PPAR) $\gamma(18)$. The upregulation of the platelet-derived growth factor/protein kinase $\mathrm{B}$ pathway partly contributes to the enhanced osteogenic potential and weakened osteoclastic differentiation of MC3T3-E1 cells, and promotes fracture healing in mice with femoral fracture following melatonin treatment (19). In addition, the BMP, extracellular regulated kinase (ERK) and Wnt signaling pathways also participate in the process, while melatonin can improve wound healing and trigger osteogenesis markers in a dose-dependent manner (20). In addition, endochondral bone formation is an important form of osteogenesis. Melatonin increases the expression of chondrogenic differentiation genes in MSCs, which could be partly blocked by luzindole (21).

Inflammation and oxidative stress are inevitable during bone injury. Melatonin is known for its potent antioxidant and anti-inflammatory properties. Oxidative stress produces reactive oxygen species (ROS) and is usually promoted by aging (22), which can lead to excessive bone resorption $(23,24)$. Melatonin is considered a potent natural antioxidant, not only due to the direct inhibition of ROS, but also due to the mobilization of the intracellular antioxidative enzyme system. Melatonin can protect MSCs against oxidation-induced apoptosis by reducing ROS production, enhancing cell viability and promoting continued differentiation (25). Melatonin can 




Figure 1. Melatonin contributes to bone repair. Melatonin reduces bone resorption by decreasing the levels of ROS and RANKL, which represent the decreased oxidative stress and osteoclastogenesis, respectively. Melatonin increases new bone regeneration by promoting the proliferation and migration, as well as the chondrogenic and osteogenic differentiation of MSCs. Melatonin also increases the level of VEGF and contributes to angiopoiesis at the site of bone injury, further promoting healing and preventing ischemic injuries. In surgeries following bone injuries, the application of melatonin helps to improve the sleep quality of patients and relieve pain. The combined application of melatonin and bone graft materials also has certain value. ROS, reactive oxygen species; RANKL, receptor activator of $\mathrm{NF}-\kappa \mathrm{B}$ ligand; MSCs, mesenchymal stem cells; VEGF, vascular endothelial growth factor.

increase solute carrier family 39 member 1 expression, activate the mitogen-activated protein kinase (MAPK)/ERK pathway, increase phosphorylated-ERK1/2/5 levels and significantly inhibit the production of ROS; moreover, zinc uptake in cells is increased (26). All the above-mentioned processes can inhibit cell apoptosis (27). In addition, melatonin is beneficial for the inhibition of oxygen free radical activity during fracture healing and the regulation of antioxidant enzyme activity, which promotes fracture healing. In a previous study, it was observed that in contrast to the fracture group, more bone binding was observed in the melatonin treatment fracture group at the same healing time (28 days after the fracture) (28).

Vascular injury often exists simultaneously in bone injuries, such as fractures, which inevitably leads to ischemia and/or hypoxia at the injury site and is extremely unfavorable for the repair of defects. Ischemia/reperfusion can cause excessive ROS production in tissues and lead to cell damage $(29,30)$. Melatonin can eliminate these adverse effects and can be used in fractures with vascular injury and compartment syndrome (31). In hypoxic environments, MC3T3-E1 cells stimulated by melatonin prefer to differentiate towards osteoblasts and promote mineralization through the p38 mitogen-activated protein kinase and protein kinase D1 pathways (32). The effect of melatonin on angiogenesis mediators also plays an important role in bone regeneration (33). Growth factors are considered potential modulators of angiogenesis. For instance, vascular endothelial growth factor (VEGF) contributes to angiogenesis (34). Melatonin treatment has been found to elevate the level of VEGF during granulation tissue formation and accelerate the angiogenic process (35), indicating that melatonin can provide beneficial effects to bone defect repair with vascular injury.

Moreover, diabetes mellitus (DM) induces high levels of ROS production. In DM model rats with fractures, the process of fiber formation and trabecular mineralization was more rapid in the melatonin treatment group compared with the control group (36).

As previously demonstrated, the use of melatonin pre-operatively helped improve the quality sleep of patients and reduced the use of opioid drugs during surgery. The normal circadian rhythm of melatonin secretion can be altered by anesthesia (37). The pre-operative use of melatonin may also have the potential to reduce the incidence of delirium; however, certain existing studies have reached varying conclusions. Al-Aama et al (38) thought exogenous melatonin administered nightly may decrease delirium occurrence in elderly medical in-patients. Sultan (39) pointed out that melatonin was successful in decreasing post-operative delirium. However, the study by de Jonghe et al (40) came to the conclusion that treatment with melatonin did not reduce the incidence of delirium in older-aged patients with hip fracture surgery. Melatonin can be used in combination with various bone graft materials to stimulate bone regeneration in large or comminuted bone injuries (41). In addition, melatonin can significantly protect bones from radiation injury and prevent epiphyseal growth plate damage (42).

The time and dose of melatonin treatment is worthy of consideration, since high doses, such as $50 \mathrm{mg} / \mathrm{kg}$, can cause decreased bone remodeling in mice, thus delaying fracture healing (14). Melatonin supplementation should also be carried 


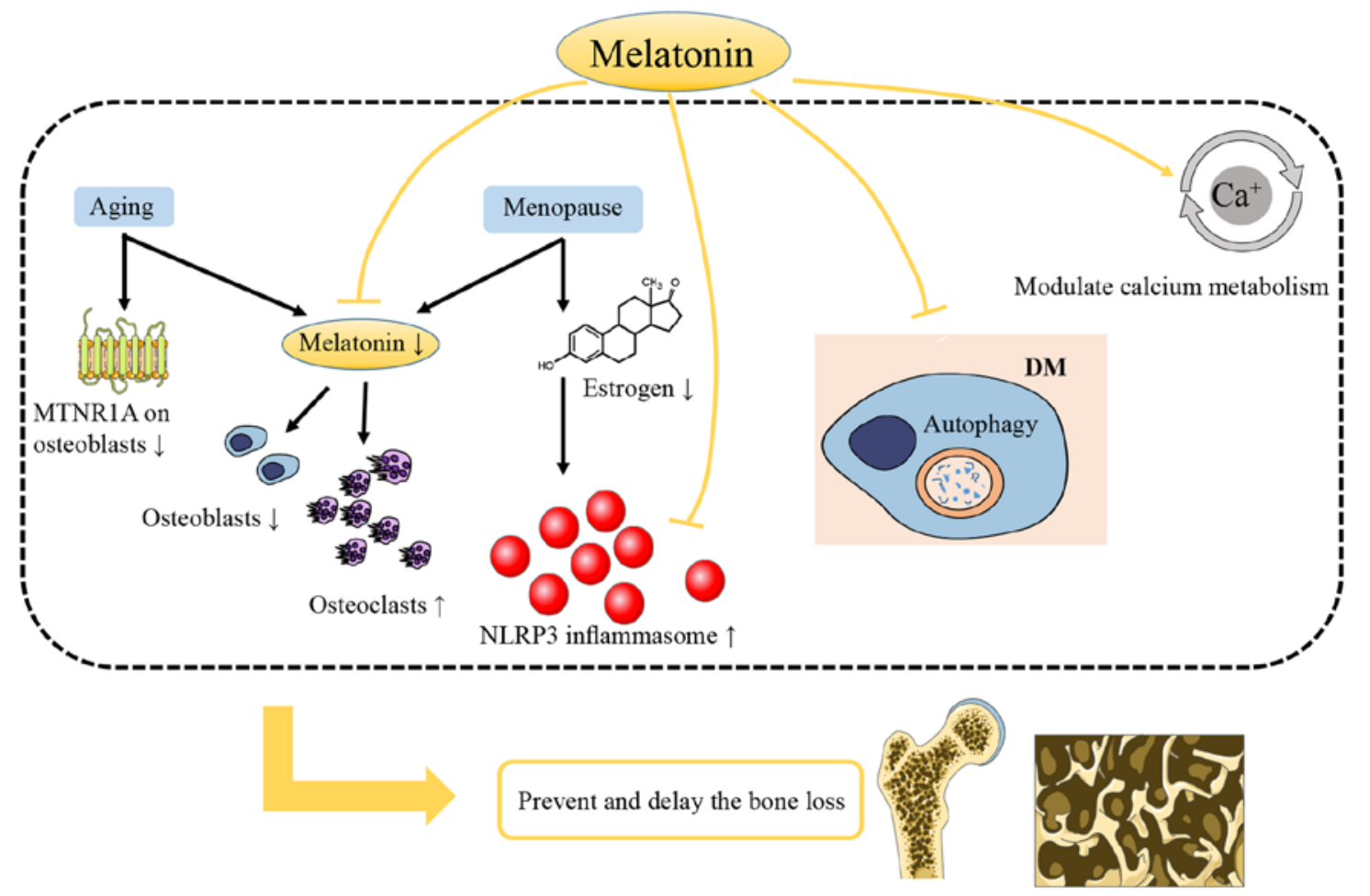

Figure 2. Positive effects of melatonin on osteoporosis. Osteoporosis often occurs in elderly and menopausal women. Melatonin production decreases in both groups and MTNR1A on the surface of osteoblasts decreases in elderly. Exogenous melatonin supplementation is effective and safe, bringing more osteoblasts and less osteoclasts. Melatonin application can reduce the high levels of the NLRP3 inflammasome in subjects suffering from estrogen deficiency. Melatonin also attenuates the autophagy of osteoblasts in patients with DM, which is considered to be beneficial in reducing bone loss. In addition, melatonin regulates calcium metabolism and prevents osteoporosis. MTNR1A, melatonin receptor 1A; NLRP3, nucleotide-binding domain and the leucine-rich repeat pyrin 3 domain; DM, diabetes mellitus.

out at night as far as possible, to adhere to its natural secretion law, to avoid breaking the normal secretory circadian rhythm, which can have adverse consequences. Evidence from the relevant animal studies is presented in Table I.

\section{Osteoporosis}

Study basics of osteoporosis. Osteoporosis is considered one of the most common diseases, and is becoming increasingly prevalent with the aging of the global population. Millions of individuals worldwide suffer from osteoporosis, particularly postmenopausal women (43-45) and the elderly $(46,47)$. The reduced bone density and damaged bone architecture caused by osteoporosis can increase the risk of fragility fractures, and lead to a higher morbidity and mortality. A variety of drugs with varying degrees of efficacy and side-effects have been used for the treatment of osteoporosis (48). Even with the existing osteoporosis treatments, the prevalence of osteoporosis is steadily increasing (49), which has created a huge economic burden to societies worldwide (50-52). Thus, novel strategies need to be developed to prevent or combat bone loss for the treatment of osteoporosis and its complications.

Potential effects of melatonin on osteoporosis. Melatonin is involved in the regulation of bone mass accumulation and loss (Fig. 2). Egermann et al (53) confirmed that the bone mass significantly decreases following pinealectomy. The decrease of melatonin secretion is associated with menopause and is one of the most important causes of osteoporosis (54). The production of melatonin decreases with age (55), which may lead to a higher bone loss among the elderly. In addition, the expression of melatonin receptor 1A (MTNR1A) on the surface of human osteoblasts decreases with age, which is more pronounced in women (15).

It has been demonstrated that melatonin supplementation can improve perimenopausal- and age-related osteoporosis. Melatonin supplementation is well-tolerated and can attenuate perimenopausal symptoms, as well as restore the balance of bone remodeling to avoid bone loss and osteoporosis (56). The daily oral melatonin administration (100 $\mathrm{mg} / \mathrm{kg}$ body weight) has been reported to increase bone formation to prevent ovariectomy-induced bone degeneration in mice (57). Although its efficacy requires further confirmation, melatonin is considered a safe nutritional supplement for peri- and postmenopausal women to improve bone density (58). Moreover, a dietary melatonin supplement has been shown to improve the microstructure and biomechanical properties of the bones of aged rats (59).

Estrogen deficiency, the major characteristic of menopause, contributes to osteoporosis. Increased levels of nucleotide-binding domain and the leucine-rich repeat pyrin 3 domain (NLRP3) inflammasome have been observed in the hippocampus of female mice with estrogen deficiency (60). Melatonin can attenuate osteoporosis induced by estrogen deficiency and can improve the osteoblastic differentiation potential by inhibiting NOD-, LRR- and pyrin domain-containing protein 3 inflammasome activation. The modulation of the $\mathrm{Wnt} / \beta$-catenin pathway is involved in this process (61). 


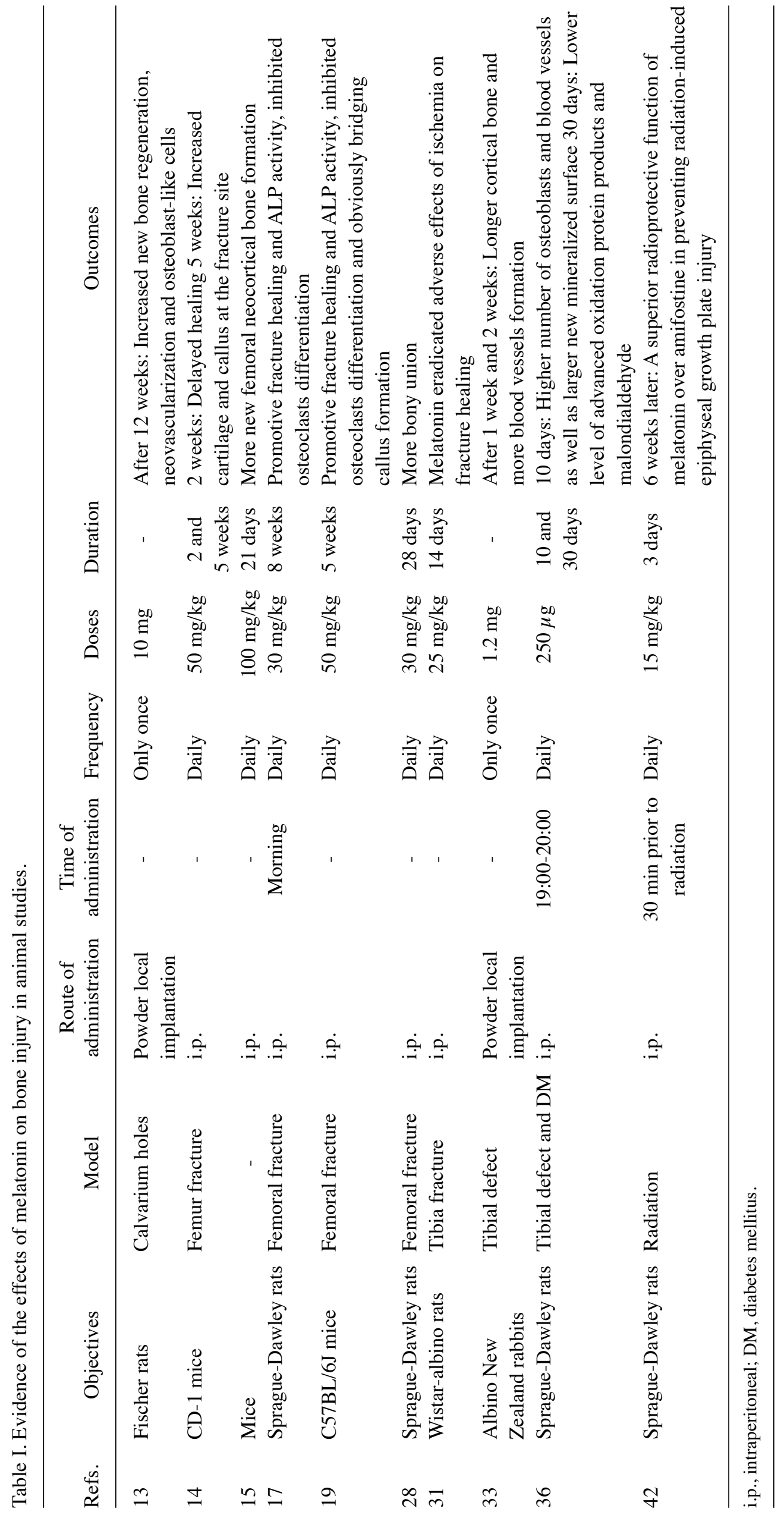


Type 2 DM and osteoporosis are both negatively affected by aging and lifestyle changes and quite often coexist. A high risk of fracture has been identified in patients with type $2 \mathrm{DM}$, particularly those with long periods of DM, poor glycemic control and diabetic complications $(62,63)$. Reduced bone remodeling is one of the characteristics of DM, and autophagy is considered to be a potential target for the management of diabetic osteoporosis $(64,65)$. The level of autophagy in osteoblasts may be reduced, and the process of DM-induced osteoporosis may be delayed by melatonin by inhibiting the ERK signaling pathway (66). Patients with multiple sclerosis (MS) have decreased serum melatonin levels and are also at risk of osteoporosis, while melatonin therapy can reduce the risk and normalize bone metabolites in MS (67). In addition to the modulation of bone formation and resorption, there are other effects of melatonin on bone metabolism, such as modulating calcium metabolism to prevent osteoporosis and hypocalcemia (68). Related evidence from relevant human and animal studies is presented in Table II.

\section{Osteoarthritis}

Basic study of OA. OA accompanied by chronic joint degeneration is one of the most common joint diseases, affecting $\sim 3.8 \%$ of the world population (69). Aging, obesity, sex, genetics, diet-related factors, specific bone/joint shapes and numerous other factors may cause the degenerative injury of articular cartilage, and reactive hyperplasia of the articular margin and subchondral bone, which may lead to the occurrence of OA (70-72).

The functions of articular cartilage depend on cartilage ECM, which primarily comprises proteoglycan and Col2 $\alpha 1$ (73). OA is characterized by the imbalance between cartilage ECM anabolism and catabolism (74). The development of OA occurs due to the presence of oxidative stress and inflammation (75). For instance, interleukin (IL)-1 $\beta$ is considered a primary inflammatory mediator in joints with local OA, and is involved in the early inflammatory process of $\mathrm{OA}$, inducing chondrocyte metabolic disorders and cartilage dysfunction, ultimately resulting in joint dysfunction $(76,77)$.

The current therapies for OA mainly focus on reducing joint load and large motion, with the aim of relieving symptoms, delaying the pathological process and improving the quality of life of patients with OA (78). Appropriate physical therapy, proper exercise, drug therapy and joint replacement surgery, among others, can be considered as treatment methods. Anti-inflammatory and analgesic drugs can be used to alleviate the symptoms of patients. However, drugs currently used in the treatment of OA, such as glucocorticoids and analgesics, have certain side-effects (79). Therefore, it is necessary to evaluate the risks of drugs and to identify novel types of low-risk drugs.

Potential effects of melatonin on OA. Inflammation plays a crucial role in the pathogenesis of OA, since mild and chronic inflammation have been shown to contribute to the symptoms and progression of OA $(80,81)$. The self-repair ability of cartilage is limited, with the cell-based articular cartilage repair ability in inflamed joints being even lower. Melatonin intervention can partly restore the chondrogenic differentiation ability of MSCs affected by IL-1 $\beta$-induced inflammation (21,77). In addition, the accumulation of ECM increases due the enhancement of ECM synthesis and the reduction of the degeneration enzyme expression induced by IL-1 $\beta$ (82). The effect of long-term intervention (21 days) is significant. Melatonin can also reduce the phosphorylation of p65 and $\mathrm{I} \kappa \mathrm{B} \alpha$, thereby inhibiting downstream NF- $\kappa \mathrm{B}$ signaling pathway activation, which plays a key role in metabolism, inflammation and apoptosis (83).

ROS can be detected in the joints of OA model rats, which can cause hyaluronic acid depolymerization and molecular configuration changes, resulting in a decrease in the viscosity of synovial fluid (84). The age-related imbalance in ROS production is responsible for cartilage degradation and chondrocyte death (85). Pro-inflammatory cytokines mediate intracellular ROS production during inflammation, impairing the viability of cells and leading to apoptosis and senescence in various cell types (86). As part of the anti-inflammatory properties of melatonin, the dynamic action of the sirtuin 1 (SIRT1) pathway is notable. Oxidative stress upregulates SIRT1 in chondrocytes, while melatonin can reduce the production of nitric oxide, cyclooxygenase-2, inducible nitric oxide synthase and prostaglandin $\mathrm{E}_{2}$ by decreasing the expression and activity of SIRT1 (87). The expression of SIRT1-dependent nuclear factor of activated T cells 5 and nicotinamide phosphoribosyltransferase in IL-1 $\beta$-stimulated chondrocytes can be suppressed by melatonin to alleviate OA (88). At the same time, melatonin also functions by activating antioxidant enzymes. Excessive ROS production reduces antioxidant enzyme expression in the progression of OA (89), while melatonin can induce the production of antioxidant enzymes, such as superoxide dismutase (90), while inhibiting ROS production (25), further suppressing oxidative stress.

The improper production of circadian clock-regulated hormones may also be involved in the occurrence of OA (91). The expression of circadian clock genes in chondrocytes is altered during the inflammatory process of OA (92), as the expression peak of brain and muscle ARNT-like 1 (Bmall) is decreased, while that of period circadian regulator 2 (Per2) is increased (93). Per 2 knockdown can reduce the expression of major cartilage degenerative enzymes, suggesting that the high expression of Per2 is one of the reasons for the progression of OA. The decline in Bmall expression is also associated with the mechanisms of OA (94), and can be restored by melatonin (95). Another study demonstrated that clock-related gene expression decreased in abnormal cartilage samples, and a nano-molar dose of melatonin restored clock-related gene expression and corrected the abnormal chondrocyte phenotype (95).

Multiple microRNAs (miRNAs/miRs) are involved in OA (96); among these, miR-140-5p has been shown to be expressed in cartilage and plays an important role in the differentiation of chondrocytes and the degeneration of cartilage (97). OA-associated cartilage changes occur in mice that lack miR-140 (98), while the overexpression of miR-140 has been shown to inhibit the synthesis of matrix catabolic enzyme (99). Elevated levels of pro-inflammatory cytokines in cartilage may reduce miR-140 expression (100). The protective roles of melatonin in OA-induced cartilage degradation are partly associated with the upregulation of miR-140 and the activation of the SMAD signaling pathways (82), which can 


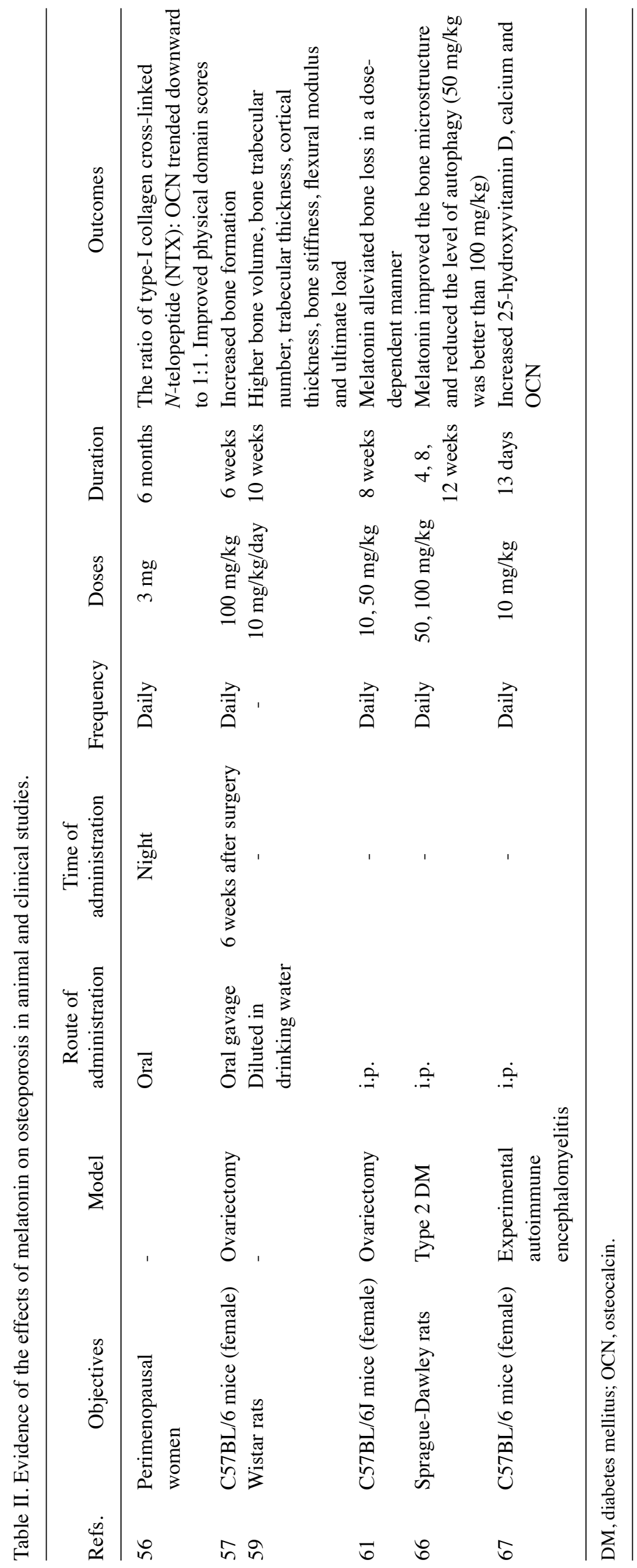


inhibit NF- $\mathrm{\kappa B}$ pathways in articular cartilage (101). In addition, other miRNAs participating in the protection of the cartilage, such as miR-526b-3p and miR-590-5p, can be upregulated by melatonin, improving the chondrogenic differentiation of MSCs (102).

An intra-articular glucocorticoid (GC) injection is a method used to alleviate inflammation and chronic pain in patients with OA. However, evidence has suggested that treatment with GCs may only be effective in the short-term, while the long-term use of GCs may not be effective, or may even aggravate cartilage degradation $(103,104)$. It has been reported that dexamethasone-induced ECM degradation in chondrocytes in a dose-dependent manner and reduced the intracellular proportion of nicotinamide adenine dinucleotide $(\mathrm{NAD})^{+} / \mathrm{NAD}+$ hydrogen $(\mathrm{NADH})$ and the supernatant concentration of NADP/NADPH. Melatonin pre-treatment can reverse these negative effects, possibly via the NAD ${ }^{+}$-dependent activation of SIRT1 (105), which can promote chondrocyte survival and ECM synthesis to prevent dexamethasone-induced damage to chondrocytes. On the other hand, NADPH oxidase has been shown to mediate the proliferation of multiple cell types, including stem cells; however, it is a major source of ROS (106). Melatonin can effectively regulate the ROS production levels of NADPH oxidase. In general, NADPH oxidase produces ROS at non-cytotoxic levels; when ROS production increases to a harmful level, excess ROS are removed by melatonin (25).

Transforming growth factor (TGF) $-\beta$ is considered to be one of the synthetic factors engaged in cartilage maintenance (107). A large amount of TGF- $\beta$ is present in healthy cartilage; however, the level of TGF- $\beta$ is decreased in OA-affected cartilage (108). Although TGF- $\beta$ exerts protective effects on cartilage, it has been proven that it can also be a destructive factor (109). Following the exogenous melatonin $(1-10 \mathrm{ng} / \mathrm{ml})$ treatment of porcine articular chondrocytes, the level of intracellular TGF- $\beta 1$ has been shown to increase. This suggests that melatonin can promote porcine chondrocyte ECM synthesis, possibly through the TGF- $\beta$ signaling pathway, and the use of melatonin instead of TGF- $\beta$ in the treatment of OA may provide a suitable amount of TGF- $\beta$ and reduce adverse reactions (110).

Melatonin can be detected in synovial fluid (111), indicating that melatonin secreted in its natural state can reach the articular cavity. The nutrition of chondrocytes in articular cartilage mainly originates from the synovial fluid, and therefore an intra-articular melatonin injection may be a potentially effective method of melatonin therapy for OA. However, melatonin treatment may only be effective in the short-term and at low concentrations. When melatonin stimulation persists, the proteolytic cleavage of RANKL proteins in the synovium is promoted, causing severe subchondral bone erosion (95). Low concentrations of melatonin, such as $1 \mathrm{nM}$, can restore Col $2 \alpha 1$ expression by inhibiting matrix metalloproteinase (MMP)-13; on the contrary, high concentrations, such as $1 \mathrm{mM}$, cannot rescue the reduced expression of Col2 21 caused by tumor necrosis factor (TNF)- $\alpha$ exposure (112). ROS generation is promoted if the concentration of melatonin is too high or the incubation time is too long (113). It has been reported that pharmacological concentrations $(\mu \mathrm{m}-\mathrm{mM})$ of melatonin promote the production of ROS and pro-inflammatory cytokines (114).
The results of the study by Liu et al (25) demonstrated that short-term melatonin treatment (5 days) promoted MSC proliferation, while long-term culture had no significant effect. These results suggested extra attention should be paid to the dose and duration of melatonin therapy, in order to avoid severe side-effects. Mild to moderate exercise can inhibit the inflammatory processes through joint compression-mediated biomechanical stimulation, helping to reduce the negative effects of high-dose and/or long-term melatonin use. There may be a synergistic effect on cartilage protection between melatonin therapy and treadmill exercise, which appears to be more effective in the early stage of the disease (112). Moreover, melatonin-combined exercise can not only restore disordered molecular clocks and correct cartilage abnormalities, but also reduce periarticular bone defects and maintain articular bone homeostasis in late-stage OA (95). However, only mild to moderate exercise is beneficial, as strenuous daily exercise aggravates OA (Fig. 3) (115). Evidence on this matter from relevant animal studies is presented in Table III.

\section{Periodontitis}

Basic study of periodontitis. Periodontitis, an inflammatory and destructive disease of the periodontal tissues, is characterized by the loss of periodontal attachment. Periodontitis is also considered as inflammation of the alveolar bone, since marginal alveolar bone loss is a key secondary feature of periodontitis, with teeth loosening and complete loss occurring at the terminal stage. The common characteristics of periodontitis usually include redness in the periodontal tissue, pain, pus overflow, halitosis and dental stone formation, which progresses to alveolar bone resorption. Dental plaque is the primary initiating factor of periodontitis, creating and maintaining an inflammatory environment in the periodontal area. Gingival inflammation (gingivitis) induced by dental plaque is the most common and mildest type of periodontal disease, with the potential to develop into periodontitis in the case of no intervention. ROS generation is another important feature of periodontitis. Oral bacteria, as well as the inflammatory and immune reaction, lead to the generation of ROS, contributing to the progression of the disease $(116,117)$.

Scaling and root planning (SRP) is considered an extremely effective basic treatment method for periodontitis. Generally, adherence to basic periodontal therapy can improve periodontal status in most patients with chronic periodontitis. However, in certain patients, progressive attachment loss cannot be terminated by SRP alone (118). For this reason, adjuvant treatment with SRP should be considered.

Potential effects of melatonin on periodontitis. Saliva and plasma melatonin levels are significantly lower in individuals with periodontal disease compared to clinically healthy subjects (119); however, the ratio of saliva and plasma melatonin levels is similar to that in healthy subjects (120). In a previous study, serum melatonin was introduced into the oral cavity through the salivary glands at a stable proportion $(\sim 33 \%$, the ratio of salivary and serum melatonin) (121). Moreover, the gingiva is one of the external sites of melatonin synthesis, and melatonin receptor 1 has been found in human gingiva, indicating that melatonin may play a receptor-mediating role 


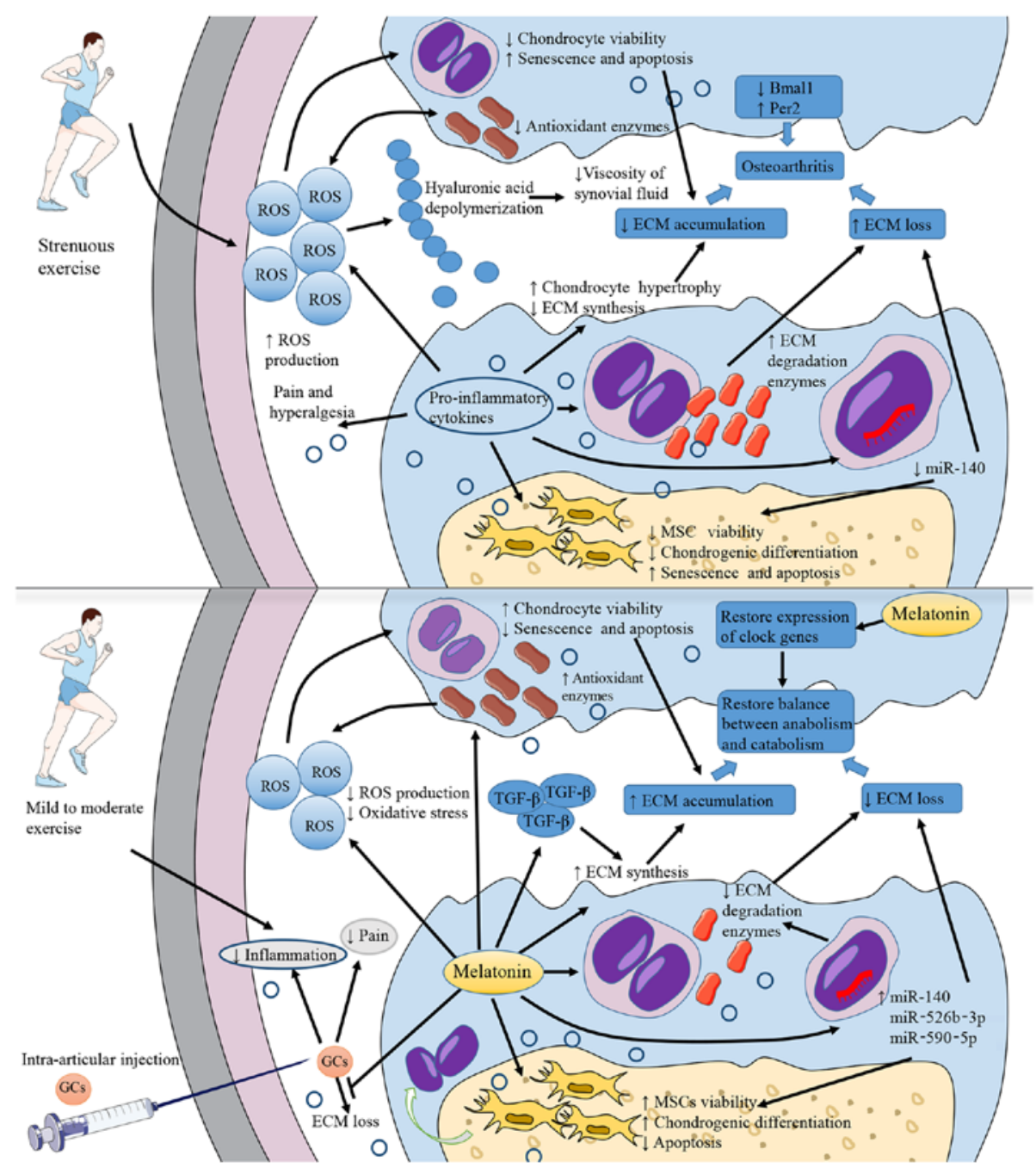

Figure 3. Pathogenesis of OA and potential roles of melatonin. The direct cause of OA is the decrease of cartilage ECM. Inflammation and strenuous exercise lead to an increased ROS production, activating oxidative stress, inhibiting chondrocytes viability, and decreasing the production of ECM components. Pro-inflammatory cytokines promote chondrocytes hypertrophy and then reduce ECM synthesis. Additionally, pro-inflammatory cytokines decrease miR-140 production in chondrocytes, inhibit the viability and chondrogenic differentiation of MSCs and increase the production of ECM degradation enzymes. All these mechanisms promote the loss of ECM. Oxidative stress also leads to decreased viscosity of synovial fluid. Pro-inflammatory cytokines are also associated with pain and hyperalgesia. The abnormal expression of clock-related genes, such as the decrease in Bmall expression and the increase in Per2 expression, is also considered to be related to the occurrence of OA. Melatonin application can effectively antagonize the above-mentioned processes, reducing the level of oxidative stress and inflammation and restoring the normal expression of clock genes. Moreover, melatonin can upregulate TGF- $\beta$ expression to increase ECM synthesis. Intra-articular injections of glucocorticoids can relieve inflammation and pain, but may also be associated with the risk of aggravating ECM loss. Melatonin can alleviate this adverse reaction. In addition, melatonin treatment combined with mild to moderate exercise may do better in OA therapeutic process. OA, osteoarthritis; ECM, extracellular matrix; ROS, reactive oxygen species; Bmall, brain and muscle ARNT-like 1; Per2, period circadian regulator 2; TGF- $\beta$, transforming growth factor $\beta$.

in the oral cavity (122). Certain studies have suggested that melatonin may serve as a potential supplementary therapy and a biomarker detecting the dynamics of periodontal disease $(123,124)$.

Melatonin levels in saliva and gingival crevicular fluid vary inversely with the severity of periodontitis $(125,126)$, suggesting that melatonin may provide protective effects against the destruction of periodontal tissues. Srinath et al (119) suggested that melatonin had antibacterial properties, since Prevotella intermedia, Streptococcus mutans and Porphyromonas gingivalis, the primary bacteria in the occurrence and progression of periodontitis, were sensitive to melatonin.

In a previous study, patients with severe periodontitis received nonsurgical periodontal therapy (NSPT) following the oral administration of melatonin at $1 \mathrm{mg} /$ day (a dietary supplement dosage advised by the Italian Ministry of Health) for 1 month (127). All patients were able to tolerate melatonin well, demonstrating a significant reduction in probing depth (PD) within 6 months, suggesting that melatonin supplementation is likely to promote the healing process of periodontal pockets following long-term treatment (127). A small number of mild adverse reactions were observed at the initial stage of oral administration, which disappeared within a few days without affecting the compliance of the patients (127). Another study reported that, when combined with NSPT, the oral administration of $2 \mathrm{mg}$ melatonin daily for 30 days improved the clinical attachment level (CAL) and PD significantly following long-term treatment (3 and 6 months) (128). Bazyar et al (129) obtained similar results with $6 \mathrm{mg}$ melatonin 


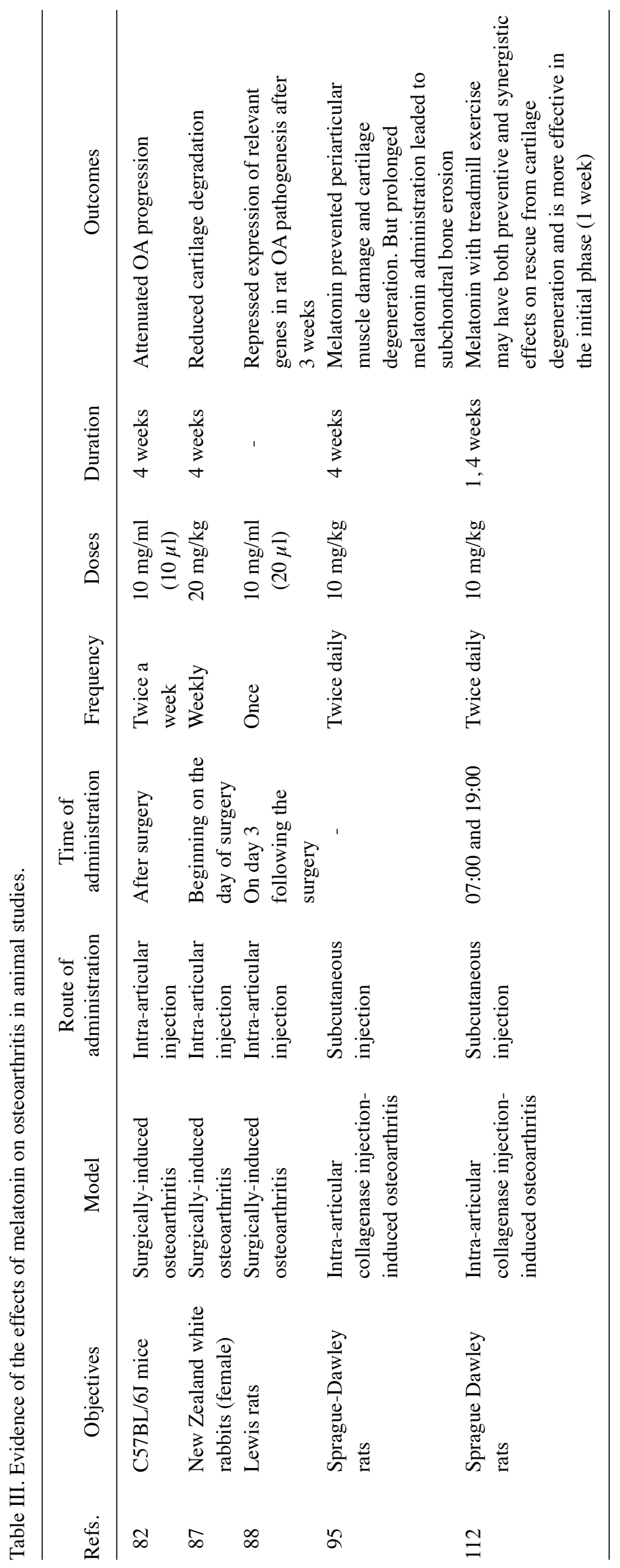


daily administration daily with NPST. In a rat model of periapical periodontitis, following an i.p. injection of melatonin (10 mg/kg) for 21 days, radiological periapical bone loss and osteoclasts were decreased, the OPG level was increased, and the IL-1 $\beta$, RANK and RANKL levels were decreased, as compared with the positive control. In addition, the bacteria localization score has been shown to be significantly lower following melatonin treatment (130). Renn et al (131) reported that preventive melatonin supplementation suppressed the Toll-like receptor 4/myeloid differentiation factor 88 pathway to inhibit the activation of pro-inflammatory cytokines and normalize the balance between RANKL and OPG, thus suppressing the progression of periodontitis.

There may be a bidirectional association between DM and periodontitis, both of which are common chronic diseases. According to previous studies, DM is considered to be a risk factor of periodontitis development, increasing the prevalence and severity and promoting the progression of periodontitis $(132,133)$. On the other hand, periodontitis may also increase the complications of DM (134). Patients with DM or periodontal disease have reduced melatonin levels in serum and saliva, and when the two diseases coexist, these levels are further reduced (135). In a previous study, in patients with mild to moderate periodontitis with DM, melatonin supplementation at $6 \mathrm{mg}$ once a day for 8 weeks clearly increased the serum melatonin levels; moreover, PD, CAL loss and the high sensitivity-C reactive protein and IL-6 levels were reduced during NSPT (129). Balci Yuce et al (136) reported that melatonin decreased osteoclasts and inhibited alveolar bone resorption in rats suffering from both DM and periodontitis; however, no decrease in bone loss was observed in rats with periodontitis alone. Oral local melatonin application was found to delay bone loss during periodontitis in patients with DM by downregulating pro-inflammatory factors $(137,138)$.

There is a clear association between periodontitis and obesity. The prevalence of periodontitis and degree of inflammation in obese or overweight patients seem to be higher compared to individuals with normal weight $(139,140)$. An experimental study reported that, when the two diseases coexisted, a significant elevation was observed in periodontal destruction, lipid dysbolism, glucose levels and hepatic damage parameters, thus revealing comorbidity effects (141). These comorbidity effects may be associated with the circadian clock (142). Considered as an important modulator of the circadian clock, melatonin may be a key mechanism in this comorbidity effect $(11,143)$.

Reduced levels of melatonin have been found to be associated with obesity (144). On the other hand, patients with periodontitis, and aggressive periodontitis in particular, are likely to have significantly lower levels of melatonin in saliva and gingival crevicular fluid $(120,123)$. Melatonin supplementation helps restore lipid and glucose metabolism, reduce pro-inflammatory factor expression, improve body weight control and avoid obesity-related complications in obese patients (144-146). Rats with obesity or periodontitis have been shown to exhibited significantly lower circulating melatonin levels, although these levels are further reduced in rats with both obesity and periodontitis (147). In addition, a markedly increased destruction of periodontal tissue was observed in rats suffering from both obesity and periodontitis, with evident inflammatory infiltration and osteoclastic activity (147). There were almost significant negative associations between circulating melatonin levels and periodontal pocket depth, dental plaque index and modified gingival index (147). Combined therapy with SRP and melatonin supplementation significantly reduced alveolar bone destruction and pro-inflammatory cytokines in rats with comorbidities of obesity and periodontitis, providing a protective effect (148). When periodontitis, pinealectomy, or a combination of both are present, the TNF and insulin concentration, as well as the homeostasis model assessment of insulin resistance index, are increased, indicating insulin resistance (149). Pineal excision can also lead to lipid profile dysregulation, which may be improved to a similar degree to the control group by melatonin alternative therapy, indicating that alternative melatonin therapy provides a therapeutic effect against dyslipidemia (149).

Periodontal ligament cells can secrete various cytokines to modulate and maintain the homeostasis of periodontal tissues, thereby playing an arrestive role in alveolar bone metabolism (150). Periodontal tissue regeneration can be enhanced by conditioned medium from periodontal ligament stem cells in a concentration-dependent manner by suppressing TNF- $\alpha$ production (151). There is an important balance between cementum formation and bone loss during the maintenance of periodontal health. Melatonin inhibited ethanol-induced ROS production and senescence-like phenotypes in human periodontal ligament stem cells and cementoblasts. In addition, it restored the decreased osteoblastic/cementoblastic differentiation, and increased osteoclastic differentiation through the protein never in mitosis gene A interacting-1 pathway. Furthermore, the downregulation of certain pathways, such as the MAPK, AMP-activated protein kinase, mammalian target of rapamycin (mTOR) and nuclear factor of activated T-cells c-1 pathways, has been suggested to exert protective effects against ethanol-induced senescence (152).

El-Sharkawy et al (153) reported that a daily dietary supplement of $10 \mathrm{mg}$ melatonin may be an effective complementary treatment for patients with insomnia with generalized chronic periodontitis, resulting in an improved CAL and sleep quality, as well as lower PD and salivary TNF- $\alpha$ levels. During the entire study period, the improvement in insomnia was maintained for up to 6 months without any rebound, even though the daily dietary melatonin supplement was administered for only 2 months. As shown by previous data, the level of systemic inflammatory markers in sleep disorders increased significantly (154). There may be a certain degree of bidirectional association between sleep disorders and periodontal disease. Therefore, improving sleep quality may itself improve the response to periodontal therapy (Fig. 4). Evidence from relevant human and animal studies on this matter are presented in Table IV.

\section{Differential effects of melatonin administered at various concentrations and times}

In the majority of previous studies, melatonin has been found to play a positive role in bone tissue and bone-related diseases. Melatonin supplementation in humans has a generally favorable safety profile. Clinical studies have demonstrated that the use of melatonin in the short-(days) and medium-term (weeks 


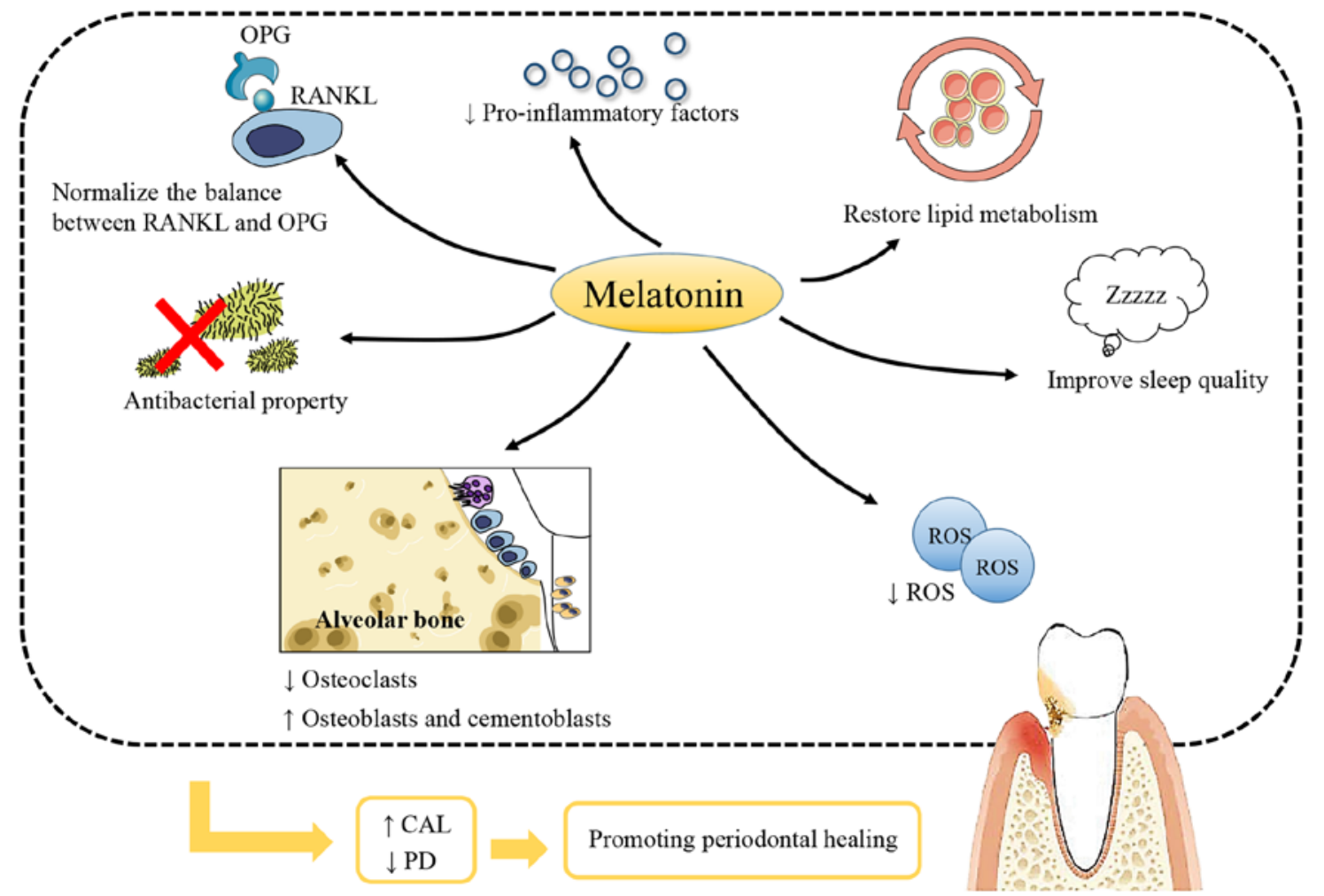

Figure 4. Melatonin promotes periodontal healing. Melatonin is considered to have the properties of antibiosis, regulating of the balance of RANKL and OPG, and reducing pro-inflammatory factors and ROS production in periodontal tissues. Melatonin cannot only reduce the number of osteoclasts and increase that of osteoblasts in alveolar bone, but can also increase that of cementoblasts. The ability of melatonin to restore lipid metabolism is also beneficial to periodontal healing because of the mutual promotion of obesity and periodontitis. In addition, the improvement of sleep quality is helpful. On the whole, melatonin application can effectively improve CAL and reduce PD, further promote periodontal healing. RANKL, receptor activator of NF- $\mathrm{KB}$ ligand; OPG, osteoprotegerin; ROS, reactive oxygen species; CAL, clinical attachment level; PD, probing depth.

to months) is safe, with only minor, transient adverse reactions reported (155). In addition to the most commonly reported adverse reactions, which are related to fatigue, mood and psychomotor or neurocognitive performance, a few studies have reported adverse events associated with endocrine and cardiovascular function, as summarized in a critical systematic review of clinical evidence (156). The safety of melatonin application in pregnant and lactating women is unknown, due to the lack of relevant research.

Although there is considerable evidence to suggest that melatonin exerts a positive effect on bone health, certain studies have reached different conclusions. Frisher et al (157) reported an association between melatonin and a significantly increased risk of fracture. In addition, in that study, it was shown that the concentration and administration time may impact the effects of melatonin treatment. As shown in Tables I-IV, the usual strategy for animal experiments is i.p. injection at doses between $10-50 \mathrm{mg} / \mathrm{kg}$. A small number of studies have used higher doses, such as $100 \mathrm{mg} / \mathrm{kg}(15,66,131)$. The intra-articular injection is a method commonly used in studies on OA, where the doses are lower $(0.1-0.2 \mu \mathrm{g})(82,88)$. In addition, the normal dose used for subcutaneous injection is $10 \mathrm{mg} / \mathrm{kg}(95,112)$. A few studies have used other methods, such as oral gavage (57), the addition of melatonin to drinking water $(59,148,149)$ and melatonin powder implantation $(13,33)$. Apart from one-off administration $(13,33)$, the shortest duration of melatonin treatment was 3 days (42), and the longest being up to 12 weeks (66). The majority of experiments used melatonin for 2-4 weeks. For clinical research, the most common route is by oral administration, with doses fluctuating between $1-10 \mathrm{mg} /$ day $(56,127-129,153)$. In addition, melatonin can be used locally in the form of $1 \%$ orabase cream for the treatment of periodontitis $(137,138)$. The drug treatment durations were $\sim 1-2$ months, and 6 months in one study (56).

To date, there is no consensus on the optimal route, dosage and time of melatonin administration. Further research is therefore required to explore the optimal route, dosage and administration time of melatonin, and determine whether long-term melatonin supplementation has any adverse effects, as well as whether melatonin can be used as a daily adjuvant in elderly, perimenopausal and postmenopausal women, and in patients with periodontitis.

\section{Conclusion and future prospects}

Melatonin is a common molecule mainly produced by the pineal gland. As an important regulatory factor of circadian rhythm, melatonin has the ability to synchronize and maintain the circadian clock in peripheral tissues with L/D cycles. In addition, melatonin is also considered cytoprotective, due to its anti-inflammatory, antitumor and antioxidant effects, and its ability to regulate hormones, the immune system and tissue regeneration.

Bone-related diseases have a high incidence, and are associated with severe and persistent symptoms, a slow recovery and a high impact on the lives of patients, as well as a heavy economic burden. The most common treatment usually exerts a curative 


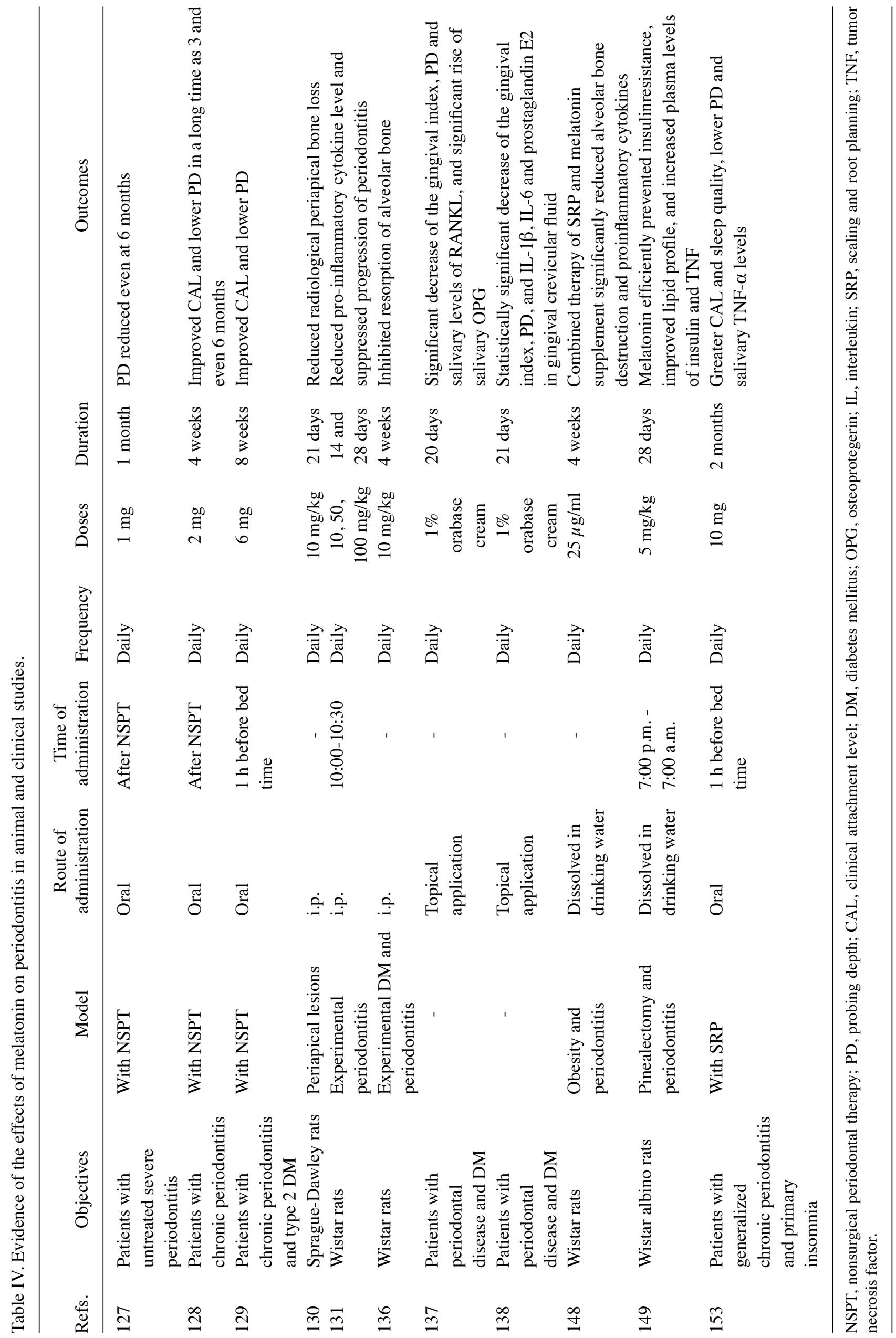




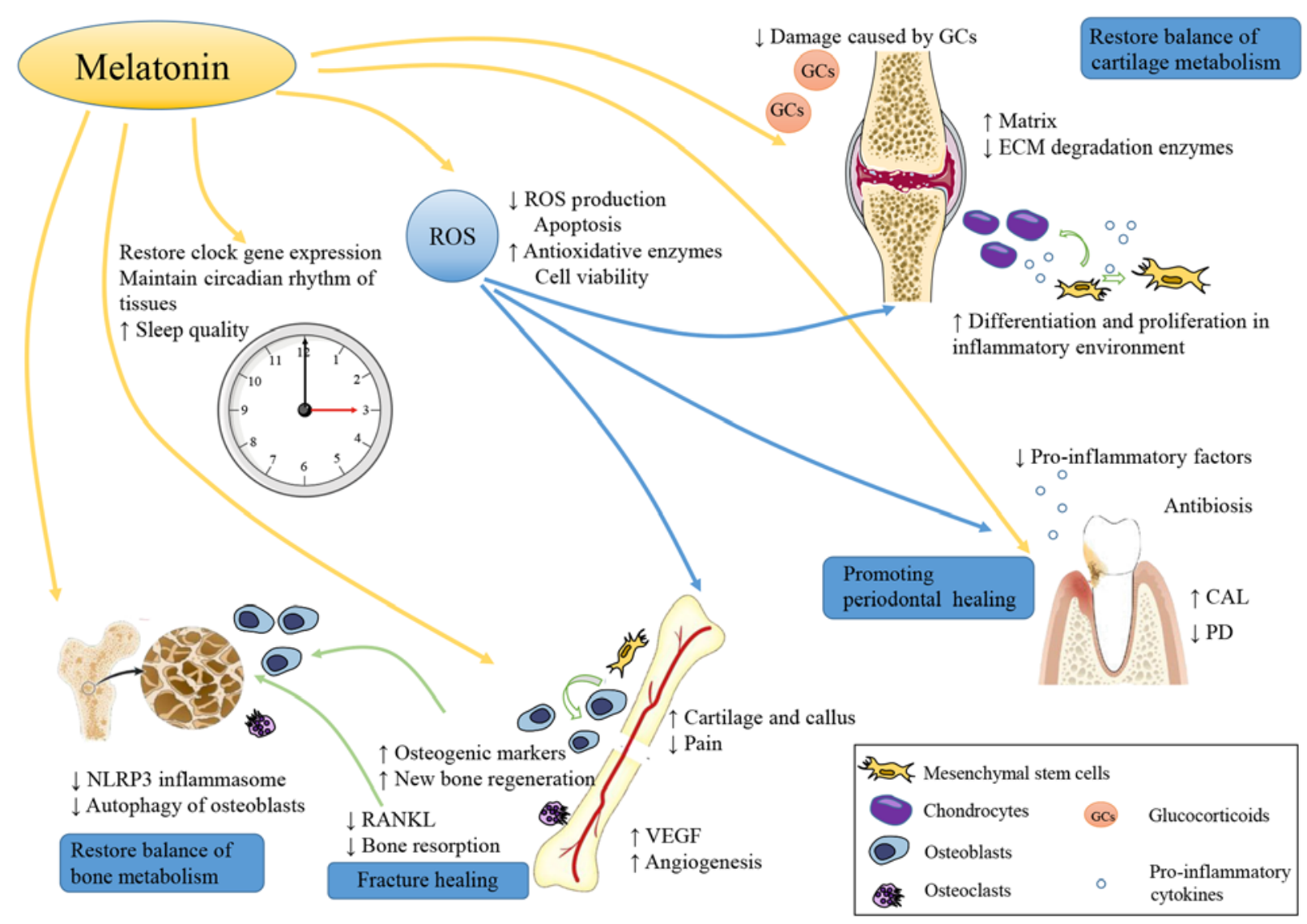

Figure 5. Roles of melatonin in bone tissue and bone-related diseases. Melatonin plays an important role in bone health. Melatonin promotes the proliferation, osteogenic and chondrogenic differentiation of MSCs, accelerating cartilage and bone formation. Moreover, melatonin inhibits osteoclasts production and prevents bone loss. The level of ROS is significantly inhibited by melatonin, which is associated with a decreased level of oxidative stress. In particular, melatonin can increase VEGF and promote angiopoiesis at the sites of bone injury, avoiding ischemic injury. The melatonin properties of reducing the level of NLRP3 inflammasome helps restore bone metabolic balance. In patients with DM, autophagy of osteoblasts is inhibited by melatonin and this effect is participated in the protection of bone health in DM patients. When used in OA, melatonin can reduce the adverse effects of intra-articular GC injection. In addition, melatonin also has an antibacterial effect on periodontitis bacteria. Metabolism of bone and cartilage is closely associated with circadian rhythm. As an important hormone regulating circadian rhythm, melatonin helps restore the circadian rhythm and this also exerts a positive effect on bone tissue and bone disease. MSCs, mesenchymal stem cells; ROS, reactive oxygen species; NLRP3, nucleotide-binding domain and the leucine-rich repeat pyrin 3 domain; VEGF, vascular endothelial growth factor; DM, diabetes mellitus; OA, osteoarthritis; GC, glucocorticoid.

effect and some side-effects. Since melatonin is inexpensive, with a wide safety margin, has a wide impact on tissues and almost no side-effects, the use of melatonin as a supplementary treatment may be a potential therapeutic option for bone disease (Fig. 5). As a widely available and versatile molecule in vivo, melatonin has potent antioxidant and anti-inflammatory properties in a variety of bone diseases. In addition, melatonin also plays a vital role in promoting osteogenesis and inhibiting osteoclastogenesis $(13,14)$. The promotion of vascularization will also provide a good boost for bone repair (31). A close association has been identified between cartilage and bone. Subchondral osteogenesis is an important type of osteogenesis (21), and cartilage and bone metabolic disorder is a common challenge in OA (72). Melatonin also protects and promotes the differentiation of cartilage (77), which is important for bone regeneration and bone development. Melatonin always drives MSCs to differentiate toward osteoblasts (17-19) and chondroblasts $(18,21,77)$ and protects MSCs from disease-induced apoptosis $(25,27,83)$. Patients with bone disease may also have other chronic metabolic diseases, such as diabetes $(62,63,132-134)$, which have adverse effects on bone health and lead to comorbidity effects. Despite these comorbidity effects, melatonin can still have beneficial effects $(66,129,136)$, which provides a novel insight for future multi-disease combination therapy.

Stomatologists pay great attention to the protection of periodontal tissue. The protective effects of melatonin on periodontal tissue are significant, helping patients with periodontitis retain more alveolar bone, providing more possibilities for natural tooth retention and subsequent tooth defect repair. In addition, the protective effects of melatonin on alveolar bone can also accelerate the process of osteointegration during implant restoration, to obtain a better implantation effect (158). Melatonin can be used to inhibit the enhancement of oxidative stress in oral tissues in the period immediately following tooth extraction to avoid excessive alveolar bone loss (159).

Human tissues are regulated by a circadian rhythm. In particular, bone metabolism is closely associated with the circadian rhythm, including the development, repair and remodeling of bone tissue; cartilage metabolism is also regulated in a similar manner (160-162). The disruption of the circadian rhythm leads to a series of adverse effects. As an important hormone regulating the circadian rhythm, melatonin helps restore the circadian rhythm (143). Sleep disorders and depression are also common symptoms among the 
elderly (163) and perimenopausal women (164). Melatonin can improve the sleep quality and mental health of patients, thus improving patient compliance; thus, the efficacy of melatonin treatment can be further consolidated.

In conclusion, melatonin functions as a protector in bone injury, osteoporosis, OA and periodontitis by exerting multiple effects. Melatonin supplementation in humans has a generally favorable safety profile. Due to the protective effects of melatonin, as well as its low price and high safety, exploring melatonin as a supplement to bone tissue and bone-related disease therapy is worthwhile. However, since a few studies have reported adverse effects, and there is no consensus on the optimal program of melatonin administration, further research is required to explore the optimal administration conditions and safety of long-term melatonin supplementation.

\section{Acknowledgements}

Not applicable.

\section{Funding}

The present study was supported by the National Natural Science Foundation of China for Distinguished Young Scholars (grant no. 31725011 to LC).

\section{Availability of data and materials}

Not applicable.

\section{Authors' contributions}

XL was involved in data curation, investigation and visualization, and in the writing of the original draft. SY was involved in the conceptualization of the study, as well as in the preparation of the figures, writing of the original draft, and in the writing, reviewing and editing of the manuscript. GC, WZ, JP and $\mathrm{XH}$ were involved in in the writing, reviewing and editing of the present review article. LC was involved in the conceptualization of the study, as well as in funding acquisition, project administration, study supervision, and in the writing, reviewing and editing of the manuscript.

\section{Ethics approval and consent to participate}

Not applicable.

\section{Patient consent for publication}

Not applicable.

\section{Competing interests}

The authors declare that they have no competing interests.

\section{References}

1. Maria S and Witt-Enderby PA: Melatonin effects on bone: Potential use for the prevention and treatment for osteopenia, osteoporosis, and periodontal disease and for use in bone-grafting procedures. J Pineal Res 56: 115-125, 2014.
2. Johnell $\mathrm{O}$ and Kanis JA: An estimate of the worldwide prevalence and disability associated with osteoporotic fractures. Osteoporos Int 17: 1726-1733, 2006.

3. Kanis JA: Diagnosis of osteoporosis and assessment of fracture risk. Lancet 359: 1929-1936, 2002.

4. Meng X, Li Y, Li S, Zhou Y, Gan RY, Xu DP and Li HB: Dietary sources and bioactivities of melatonin. Nutrients 9: 367, 2017.

5. Cipolla-Neto J and Amaral FGD: Melatonin as a hormone: New physiological and clinical insights. Endocr Rev 39: 990-1028, 2018.

6. Reiter RJ: Pineal melatonin: Cell biology of its synthesis and of its physiological interactions. Endocr Rev 12: 151-180, 1991.

7. Amaral FGD and Cipolla-Neto J: A brief review about melatonin, a pineal hormone. Arch Endocrinol Metab 62: 472-479, 2018

8. Simonneaux V and Ribelayga C: Generation of the melatonin endocrine message in mammals: A review of the complex regulation of melatonin synthesis by norepinephrine, peptides, and other pineal transmitters. Pharmacol Rev 55: 325-395, 2003.

9. Tan DX, Manchester LC, Hardeland R, Lopez-Burillo S, Mayo JC, Sainz RM and Reiter RJ: Melatonin: A hormone, a tissue factor, an autocoid, a paracoid, and an antioxidant vitamin. J Pineal Res 34: 75-78, 2003.

10. Permuy M, López-Peña M, González-Cantalapiedra A and Muñoz F: Melatonin: A review of its potential functions and effects on dental diseases. Int J Mol Sci 18: 865, 2017.

11. Tordjman S, Chokron S, Delorme R, Charrier A, Bellissant E, Jaafari N and Fougerou C: Melatonin: Pharmacology, functions and therapeutic benefits. Curr Neuropharmacol 15: 434-443, 2017.

12. Nauth A, Schemitsch E, Norris B, Nollin Z and Watson JT: Critical-size bone defects: Is there a consensus for diagnosis and treatment? J Orthop Trauma 32 (Suppl 1): S7-S11, 2018.

13. Shino H, Hasuike A, Arai Y, Honda M, Isokawa K and Sato S: Melatonin enhances vertical bone augmentation in rat calvaria secluded spaces. Med Oral Patol Oral Cir Bucal 21: e122-e126, 2016.

14. Histing T, Anton C, Scheuer C, Garcia P, Holstein JH, Klein M, Matthys R, Pohlemann T and Menger MD: Melatonin impairs fracture healing by suppressing RANKL-mediated bone remodeling. J Surg Res 173: 83-90, 2012.

15. Satomura K, Tobiume S, Tokuyama R, Yamasaki Y, Kudoh K, Maeda E and Nagayama M: Melatonin at pharmacological doses enhances human osteoblastic differentiation in vitro and promotes mouse cortical bone formation in vivo. J Pineal Res 42 : 231-239, 2007.

16. Sethi S, Radio NM, Kotlarczyk MP, Chen CT, Wei YH, Jockers R and Witt-Enderby PA: Determination of the minimal melatonin exposure required to induce osteoblast differentiation from human mesenchymal stem cells and these effects on downstream signaling pathways. J Pineal Res 49: 222-238, 2010.

17. Dong P, Gu X, Zhu G, Li M, Ma B and Zi Y: Melatonin induces osteoblastic differentiation of mesenchymal stem cells and promotes fracture healing in a rat model of femoral fracture via neuropeptide Y/neuropeptide $\mathrm{Y}$ receptor Y1 signaling. Pharmacology 102: 272-280, 2018

18. Luchetti F, Canonico B, Bartolini D, Arcangeletti M, Ciffolilli S, Murdolo G, Piroddi M, Papa S, Reiter RJ and Galli F: Melatonin regulates mesenchymal stem cell differentiation: A review. J Pineal Res 56: 382-397, 2014.

19. Zhu G, Ma B, Dong P, Shang J, Gu X and Zi Y: Melatonin promotes osteoblastic differentiation and regulates PDGF/AKT signaling pathway. Cell Biol Int 44: 402-411, 2020.

20. Park KH, Kang JW, Lee EM, Kim JS, Rhee YH, Kim M, Jeong SJ, Park YG and Kim SH: Melatonin promotes osteoblastic differentiation through the BMP/ERK/Wnt signaling pathways. J Pineal Res 51: 187-194, 2011.

21. Gao W, Lin M, Liang A, Zhang L, Chen C, Liang G, Xu C, Peng Y, Chen C, Huang D and Su P: Melatonin enhances chondrogenic differentiation of human mesenchymal stem cells. J Pineal Res 56: 62-70, 2014.

22. Zhang B, Bailey WM, McVicar AL and Gensel JC: Age increases reactive oxygen species production in macrophages and potentiates oxidative damage after spinal cord injury. Neurobiol Aging 47: 157-167, 2016.

23. Fraser JH, Helfrich MH, Wallace HM and Ralston SH: Hydrogen peroxide, but not superoxide, stimulates bone resorption in mouse calvariae. Bone 19: 223-226, 1996

24. Lee NK, Choi YG, Baik JY, Han SY, Jeong DW, Bae YS, Kim N and Lee SY: A crucial role for reactive oxygen species in RANKL-induced osteoclast differentiation. Blood 106: 852-859, 2005 . 
25. Liu X, Gong Y, Xiong K, Ye Y, Xiong Y, Zhuang Z, Luo Y, Jiang $Q$ and He F: Melatonin mediates protective effects on inflammatory response induced by interleukin-1 beta in human mesenchymal stem cells. J Pineal Res 55: 14-25, 2013.

26. Liu XW, Zi Y, Liu YE, Zhang YB, Xiang LB and Hou MX: Melatonin exerts protective effect on N2a cells under hypoxia conditions through Zip1/ERK pathway. Neurosci Lett 595: 74-80, 2015.

27. Xu S, Yang Y, Han S and Wu Z: ZIP1 and zinc inhibits fluoride-induced apoptosis in MC3T3-E1 cells. Biol Trace Elem Res 159: 399-409, 2014.

28. Halıcı M, Öner M, Güney A, Canöz Ö, Narin F and Halıcı C: Melatonin promotes fracture healing in the rat model. Eklem Hastalik Cerrahisi 21: 172-177, 2010.

29. Quesnelle KM, Bystrom PV and Toledo-Pereyra LH: Molecular responses to ischemia and reperfusion in the liver. Arch Toxicol 89: 651-657, 2015.

30. Bagheri F, Khori V, Alizadeh AM, Khalighfard S, Khodayari S and Khodayari H: Reactive oxygen species-mediated cardiac-reperfusion injury: Mechanisms and therapies. Life Sci 165: 43-55, 2016.

31. Erdem M, Gulabi D, Asci M,Bostan B, Gunes T and Koseoglu RD The effects of melatonin and caffeic acid phenethyl ester (CAPE) on fracture healing under ischemic conditions. Acta Orthop Traumatol Turc 48: 339-345, 2014

32. Son JH, Cho YC, Sung IY, Kim IR, Park BS and Kim YD Melatonin promotes osteoblast differentiation and mineralization of MC3T3-E1 cells under hypoxic conditions through activation of PKD/p38 pathways. J Pineal Res 57: 385-392, 2014.

33. Ramírez-Fernández MP, Calvo-Guirado JL, de-Val JE, Delgado-Ruiz RA, Negri B, Pardo-Zamora G, Peñarrocha D, Barona C, Granero JM and Alcaraz-Baños M: Melatonin promotes angiogenesis during repair of bone defects: A radiological and histomorphometric study in rabbit tibiae. Clin Oral Investig 17: 147-158, 2013.

34. Melincovici CS, Boşca AB, Susman S, Mărginean M, Mihu C, Istrate M, Moldovan IM, Roman AL and Mihu CM: Vascular endothelial growth factor (VEGF)-key factor in normal and pathological angiogenesis. Rom J Morphol Embryol 59: 455-467, 2018.

35. Pugazhenthi K, Kapoor M, Clarkson AN, Hall I and Appleton I: Melatonin accelerates the process of wound repair in full-thickness incisional wounds. J Pineal Res 44: 387-396, 2008.

36. Yildirimturk S, Batu S, Alatli C, Olgac V, Firat D and Sirin Y: The effects of supplemental melatonin administration on the healing of bone defects in streptozotocin-induced diabetic rats J Appl Oral Sci 24: 239-249, 2016.

37. de Carvalho Nogueira EF, de Oliveira Vasconcelos R, Teixeira Correia SS, Souza Catunda I, Amorim JA and do Egito Cavalcanti Vasconcelos B: Is there a benefit to the use of melatonin in preoperative zygomatic fractures? J Oral Maxillofac Surg 77: 2017.e1-2017.e7, 2019.

38. Al-Aama T, Brymer C, Gutmanis I, Woolmore-Goodwin SM, Esbaugh J and Dasgupta M: Melatonin decreases delirium in elderly patients: A randomized, placebo-controlled trial. Int J Geriatr Psychiatry 26: 687-694, 2011.

39. Sultan SS: Assessment of role of perioperative melatonin in prevention and treatment of postoperative delirium after hip arthroplasty under spinal anesthesia in the elderly. Saudi J Anaesth 4: 169-173, 2010

40. de Jonghe A, van Munster BC, Goslings JC, Kloen P, van Rees C, Wolvius R, van Velde R, Levi M, de Haan RJ, de Rooij SE and Amsterdam Delirium Study Group: Effect of melatonin on incidence of delirium among patients with hip fracture: A multicentre, double-blind randomized controlled trial. CMAJ 186 E547-E556, 2014.

41. Majidinia M, Reiter RJ, Shakouri SK, Mohebbi I, Rastegar M, Kaviani M, Darband SG, Jahanban-Esfahlan R, Nabavi SM and Yousefi B: The multiple functions of melatonin in regenerative medicine. Ageing Res Rev 45: 33-52, 2018.

42. Topkan E, Tufan H, Yavuz AA, Bacanli D, Onal C, Kosdak S and Yavuz MN: Comparison of the protective effects of melatonin and amifostine on radiation-induced epiphyseal injury. Int J Radiat Biol 84: 796-802, 2008.

43. Rachner TD, Khosla S and Hofbauer LC: Osteoporosis: Now and the future. Lancet 377: 1276-1287, 2011.

44. Cipriani C, Pepe J, Bertoldo F, Bianchi G, Cantatore FP, Corrado A, Di Stefano M, Frediani B, Gatti D and Giustina A, et al: The epidemiology of osteoporosis in Italian postmenopausa women according to the National Bone Health Alliance (NBHA) diagnostic criteria: A multicenter cohort study. J Endocrinol Invest 41: 431-438, 2018.
45. Parizad N, Baghi V, Karimi EB and Ghanei Gheshlagh R: The prevalence of osteoporosis among Iranian postmenopausal women with type 2 diabetes: A systematic review and meta-analysis. Diabetes Metab Syndr 13: 2607-2612, 2019.

46. Melton LJ III: The prevalence of osteoporosis: Gender and racial comparison. Calcif Tissue Int 69: 179-181, 2001.

47. Coughlan T and Dockery F: Osteoporosis and fracture risk in older people. Clin Med (Lond) 14: 187-191, 2014.

48. Liu GF, Wang ZQ, Liu L, Zhang BT, Miao YY and Yu SN: A network meta-analysis on the short-term efficacy and adverse events of different anti-osteoporosis drugs for the treatment of postmenopausal osteoporosis. J Cell Biochem 119: 4469-4481, 2018.

49. Cui Z, Meng X, Feng H, Zhuang S, Liu Z, Zhu T, Ye K, Xing Y, Sun C, Zhou F and Tian Y: Estimation and projection about the standardized prevalence of osteoporosis in mainland China. Arch Osteoporos 15: 2, 2019.

50. Lane NE: Epidemiology, etiology, and diagnosis of osteoporosis. Am J Obstet Gynecol 194 (2 Suppl): S3-S11, 2006.

51. Mohd-Tahir NA and Li SC: Economic burden of osteoporosis-related hip fracture in Asia: A systematic review. Osteoporos Int 28: 2035-2044, 2017.

52. Hopkins RB, Burke N, Von Keyserlingk C, Leslie WD, Morin SN, Adachi JD, Papaioannou A, Bessette L, Brown JP, Pericleous L and Tarride J: The current economic burden of illness of osteoporosis in Canada. Osteoporos Int 27: 3023-3032, 2016.

53. Egermann M, Gerhardt C, Barth A, Maestroni GJ, Schneider E and Alini M: Pinealectomy affects bone mineral density and structure-an experimental study in sheep. BMC Musculoskelet Disord 12: 271, 2011.

54. Pines A: Circadian rhythm and menopause. Climacteric 19: 551-552, 2016.

55. Sack RL, Lewy AJ, Erb DL, Vollmer WM and Singer CM: Human melatonin production decreases with age. J Pineal Res 3: 379-388, 1986

56. Kotlarczyk MP, Lassila HC, O'Neil CK, D'Amico F, Enderby LT, Witt-Enderby PA and Balk JL: Melatonin osteoporosis prevention study (MOPS): A randomized, double-blind, placebo-controlled study examining the effects of melatonin on bone health and quality of life in perimenopausal women. J Pineal Res 52: 414-426, 2012.

57. Sharan K, Lewis K, Furukawa T and Yadav VK: Regulation of bone mass through pineal-derived melatonin-MT2 receptor pathway. J Pineal Res 63: e12423, 2017.

58. Bao T, Zeng L, Yang K, Li Y, Ren F, Zhang Y and Gao Z: Can melatonin improve the osteopenia of perimenopausal and postmenopausal women? A meta-analysis. Int J Endocrinol 2019: $5151678,2019$.

59. Tresguerres IF, Tamimi F, Eimar H, Barralet JE, Prieto S, Torres J, Calvo-Guirado JL and Tresguerres JA: Melatonin dietary supplement as an anti-aging therapy for age-related bone loss. Rejuvenation Res 17: 341-346, 2014.

60. Xu Y, Sheng H, Bao Q, Wang Y, Lu J and Ni X: NLRP3 inflammasome activation mediates estrogen deficiency-induced depression- and anxiety-like behavior and hippocampal inflammation in mice. Brain Behav Immun 56: 175-186, 2016.

61. Xu L, Zhang L, Wang Z, Li C, Li S, Li L, Fan Q and Zheng L: Melatonin suppresses estrogen deficiency-induced osteoporosis and promotes osteoblastogenesis by inactivating the NLRP3 inflammasome. Calcif Tissue Int 103: 400-410, 2018.

62. Si Y, Wang C, Guo Y, Xu G and Ma Y: Prevalence of osteoporosis in patients with type 2 diabetes mellitus in the Chinese mainland: A systematic review and meta-analysis. Iran J Public Health 48: 1203-1214, 2019

63. Paschou SA, Dede AD, Anagnostis PG, Vryonidou A, Morganstein D and Goulis DG: Type 2 diabetes and osteoporosis: A guide to optimal management. J Clin Endocrinol Metab 102: 3621-3634, 2017.

64. Lecka-Czernik B: Diabetes, bone and glucose-lowering agents: Basic biology. Diabetologia 60: 1163-1169, 2017.

65. Meng HZ, Zhang WL, Liu F and Yang MW: Advanced glycation end products affect osteoblast proliferation and function by modulating autophagy via the receptor of advanced glycation end products/raf protein/mitogen-activated protein kinase/extracellular signal-regulated kinase kinase/extracellular signal-regulated kinase (RAGE/Raf/MEK/ERK) pathway. J Biol Chem 290: 28189-28199, 2015.

66. Zhang WL, Meng HZ, Yang RF, Yang MW, Sun GH, Liu JH, Shi PX, Liu F and Yang B: Melatonin suppresses autophagy in type 2 diabetic osteoporosis. Oncotarget 7: 52179-52194, 2016. 
67. Ghareghani M, Scavo L, Arnoult D, Zibara K and Farhadi N Melatonin therapy reduces the risk of osteoporosis and normalizes bone formation in multiple sclerosis. Fundam Clin Pharmacol 32: 181-187, 2018

68. Witt-Enderby PA, Radio NM, Doctor JS and Davis VL: Therapeutic treatments potentially mediated by melatonin receptors: Potential clinical uses in the prevention of osteoporosis cancer and as an adjuvant therapy. J Pineal Res 41: 297-305, 2006.

69. March L, Smith EU, Hoy DG, Cross MJ, Sanchez-Riera L, Blyth F, Buchbinder R, Vos T and Woolf AD: Burden of disability due to musculoskeletal (MSK) disorders. Best Pract Res Clin Rheumatol 28: 353-366, 2014.

70. Neogi T and Zhang Y: Epidemiology of osteoarthritis. Rheum Dis Clin North Am 39: 1-19, 2013.

71. Thomas AC, Hubbard-Turner T, Wikstrom EA and Palmieri-Smith RM: Epidemiology of Posttraumatic Osteoarthritis. J Athl Train 52: 491-496, 2017.

72. Vina ER and Kwoh CK: Epidemiology of osteoarthritis: Literature update. Curr Opin Rheumatol 30: 160-167, 2018.

73. Gentili $\mathrm{C}$ and Cancedda R: Cartilage and bone extracellular matrix. Curr Pharm Des 15: 1334-1348, 2009.

74. Mobasheri A, Rayman MP, Gualillo O, Sellam J, van der Kraan P and Fearon U: The role of metabolism in the pathogenesis of osteoarthritis. Nat Rev Rheumatol 13: 302-311, 2017.

75. Buckwalter JA, Anderson DD, Brown TD, Tochigi Y and Martin JA: The roles of mechanical stresses in the pathogenesis of osteoarthritis: Implications for treatment of joint injuries. Cartilage 4: 286-294, 2013

76. Kapoor M, Martel-Pelletier J, Lajeunesse D, Pelletier JP and Fahmi H: Role of proinflammatory cytokines in the pathophysiology of osteoarthritis. Nat Rev Rheumatol 7: 33-42, 2011.

77. Gao B, Gao W, Wu Z, Zhou T, Qiu X, Wang X, Lian C, Peng Y, Liang A, Qiu J, et al: Melatonin rescued interleukin $1 \beta$-impaired chondrogenesis of human mesenchymal stem cells. Stem Cell Res Ther 9: 162, 2018.

78. Taruc-Uy RL and Lynch SA: Diagnosis and treatment of osteoarthritis. Prim Care 40: 821-836, vii, 2013.

79. Hainque B, Dominice J, Jaffray P, Ronot X and Adolphe M: Effects of dexamethasone on the growth of cultured rabbit articular chondrocytes: Relation with the nuclear glucocorticoid-receptor complex. Ann Rheum Dis 46: 146-152, 1987.

80. Robinson WH, Lepus CM, Wang Q, Raghu H, Mao R Lindstrom TM and Sokolove J: Low-grade inflammation as a key mediator of the pathogenesis of osteoarthritis. Nat Rev Rheumatol 12: 580-592,2016.

81. Liu-Bryan R and Terkeltaub R: Emerging regulators of the inflammatory process in osteoarthritis. Nat Rev Rheumatol 11: 35-44, 2015

82. Zhang Y, Lin J, Zhou X, Chen X, Chen AC, Pi B, Pan G, Pei M, Yang H,Liu T and He F: Melatonin prevents osteoarthritis-induced cartilage degradation via targeting MicroRNA-140. Oxid Med Cell Longev 2019: 9705929, 2019.

83. Hosseinzadeh A, Kamrava SK, Joghataei MT, Darabi R, Shakeri-Zadeh A, Shahriari M, Reiter RJ, Ghaznavi H and Mehrzadi S: Apoptosis signaling pathways in osteoarthritis and possible protective role of melatonin. J Pineal Res 61: 411-425, 2016

84. Milam SB, Zardeneta G and Schmitz JP: Oxidative stress and degenerative temporomandibular joint disease: A proposed hypothesis. J Oral Maxillofac Surg 56: 214-223, 1998.

85. Bolduc JA, Collins JA and Loeser RF: Reactive oxygen species, aging and articular cartilage homeostasis. Free Radic Bio Med 132: 73-82, 2019.

86. Bakker AD, Silva VC, Krishnan R, Bacabac RG, Blaauboer ME, Lin YC, Marcantonio RA, Cirelli JA and Klein-Nulend J: Tumor necrosis factor alpha and interleukin-lbeta modulate calcium and nitric oxide signaling in mechanically stimulated osteocytes. Arthritis Rheum 60: 3336-3345, 2009.

87. Lim HD, Kim YS, Ko SH, Yoon IJ, Cho SG, Chun YH, Choi BJ and Kim EC: Cytoprotective and anti-inflammatory effects of melatonin in hydrogen peroxide-stimulated CHON-001 human chondrocyte cell line and rabbit model of osteoarthritis via the SIRT1 pathway. J Pineal Res 53: 225-237, 2012.

88. Guo JY, Li F, Wen YB, Cui HX, Guo ML, Zhang L, Zhang YF, Guo YJ and Guo YX: Melatonin inhibits Sirt1-dependent NAMPT and NFAT5 signaling in chondrocytes to attenuate osteoarthritis. Oncotarget 8: 55967-55983, 2017.

89. Cuzzocrea S, Zingarelli B, Gilad E, Hake P, Salzman AL and Szabó C: Protective effect of melatonin in carrageenan-induced models of local inflammation: Relationship to its inhibitory effect on nitric oxide production and its peroxynitrite scavenging activity. J Pineal Res 23: 106-116, 1997.
90. Ozturk G, Coşkun S, Erbaş D and Hasanoglu E: The effect of melatonin on liver superoxide dismutase activity, serum nitrate and thyroid hormone levels. Jpn J Physiol 50: 149-153, 2000.

91. Hossain FM, Hong Y, Jin Y, Choi J and Hong Y: Physiological and pathological role of circadian hormones in osteoarthritis: Dose-dependent or time-dependent? J Clin Med 8: 1415, 2019.

92.Jahanban-Esfahlan R, Mehrzadi S, Reiter RJ, Seidi K Majidinia M, Baghi HB, Khatami N, Yousefi B and Sadeghpour A: Melatonin in regulation of inflammatory pathways in rheumatoid arthritis and osteoarthritis: Involvement of circadian clock genes. Br J Pharmacol 175: 3230-3238, 2018.

93. Rong J, Zhu M, Munro J, Cornish J, McCarthy GM, Dalbeth N and Poulsen RC: Altered expression of the core circadian clock component PERIOD2 contributes to osteoarthritis-like changes in chondrocyte activity. Chronobiol Int 36: 319-331, 2019.

94. Yang W, Kang X, Liu J, Li H, Ma Z, Jin X, Qian Z, Xie T, Qin N, Feng D, et al: Clock gene Bmall modulates human cartilage gene expression by crosstalk with Sirt1. Endocrinology 157: 3096-3107, 2016.

95. Hong Y, Kim H, Lee S, Jin Y, Choi J, Lee SR, Chang KT and Hong Y: Role of melatonin combined with exercise as a switch-like regulator for circadian behavior in advanced osteoarthritic knee. Oncotarget 8: 97633-97647, 2017.

96. Nugent M: MicroRNAs: Exploring new horizons in osteoarthritis. Osteoarthritis Cartilage 24: 573-580, 2016

97. Miyaki S and Asahara H: Macro view of microRNA function in osteoarthritis. Nat Rev Rheumatol 8: 543-552, 2012.

98. Miyaki S, Sato T, Inoue A, Otsuki S, Ito Y, Yokoyama S, Kato Y, Takemoto F, Nakasa T, Yamashita S, et al: MicroRNA-140 plays dual roles in both cartilage development and homeostasis. Genes Dev 24: 1173-1185, 2010.

99. Si HB, Zeng Y, Liu SY, Zhou ZK, Chen YN, Cheng JQ, Lu YR and Shen B: Intra-articular injection of microRNA-140 (miRNA-140) alleviates osteoarthritis (OA) progression by modulating extracellular matrix (ECM) homeostasis in rats. Osteoarthritis Cartilage 25: 1698-1707, 2017.

100. Miyaki S, Nakasa T, Otsuki S, Grogan SP, Higashiyama R, Inoue $\mathrm{A}$, Kato $\mathrm{Y}$, Sato $\mathrm{T}$, Lotz $\mathrm{MK}$ and Asahara $\mathrm{H}$ : MicroRNA-140 is expressed in differentiated human articular chondrocytes and modulates interleukin-1 responses. Arthritis Rheum 60: 2723-2730, 2009.

101. Karlsen TA, de Souza GA, Ødegaard B, Engebretsen L and Brinchmann JE: microRNA-140 inhibits inflammation and stimulates chondrogenesis in a model of interleukin $1 \beta$-induced osteoarthritis. Mol Ther Nucleic Acids 5: e373, 2016.

102. Wu Z, Qiu X, Gao B, Lian C, Peng Y, Liang A, Xu C, Gao W Zhang L, Su P, et al: Melatonin-mediated miR-526b-3p and miR-590-5p upregulation promotes chondrogenic differentiation of human mesenchymal stem cells. J Pineal Res 65: e12483, 2018.

103. Jüni P, Hari R, Rutjes AW, Fischer R, Silletta MG, Reichenbach S and da Costa BR: Intra-articular corticosteroid for knee osteoarthritis. Cochrane Database Syst Rev CD005328, 2015.

104. McAlindon TE, LaValley MP, Harvey WF, Price LL, Driban JB, Zhang $\mathrm{M}$ and Ward RJ: Effect of intra-articular triamcinolone vs saline on knee cartilage volume and pain in patients with knee osteoarthritis: A randomized clinical trial. JAMA 317: 1967-1975, 2017

105. Yang W, Kang X, Qin N, Li F, Jin X, Ma Z, Qian Z and Wu S: Melatonin protects chondrocytes from impairment induced by glucocorticoids via NAD ${ }^{+}$-dependent SIRT1. Steroids 126 : 24-29, 2017.

106. Garrido-Urbani S, Jaquet V and Imhof BA: ROS and NADPH oxidase: Key regulators of tumor vascularisation. Med Sci (Paris) 30: 415-421, 2014 (In French).

107. Ahn J, Kim SA, Kim KW, Oh JH and Kim SJ: Optimization of TGF- $\beta 1$-transduced chondrocytes for cartilage regeneration in a 3D printed knee joint model. PLoS One 14: e0217601, 2019.

108. Verdier MP, Seité S, Guntzer K, Pujol JP and Boumédiène K: Immunohistochemical analysis of transforming growth factor beta isoforms and their receptors in human cartilage from normal and osteoarthritic femoral heads. Rheumatol Int 25: $118-124,2005$

109. Fang J, Xu L, Li Y and Zhao Z: Roles of TGF-beta 1 signaling in the development of osteoarthritis. Histol Histopathol 31: 1161-1167, 2016.

110. Pei M, He F, Wei L and Rawson A: Melatonin enhances cartilage matrix synthesis by porcine articular chondrocytes. J Pineal Res 46: 181-187, 2009. 
111. Maestroni GJ, Sulli A, Pizzorni C, Villaggio B and Cutolo M: Melatonin in rheumatoid arthritis: Synovial macrophages show melatonin receptors. Ann N Y Acad Sci 966: 271-275, 2002.

112. Hong Y, Kim H, Lee Y, Lee S, Kim K, Jin Y, Lee SR, Chang KT and Hong Y: Salutary effects of melatonin combined with treadmill exercise on cartilage damage. J Pineal Res 57: 53-66, 2014

113. Osseni RA, Rat P, Bogdan A, Warnet JM and Touitou Y: Evidence of prooxidant and antioxidant action of melatonin on human liver cell line HepG2. Life Sci 68: 387-399, 2000.

114. Zhang HM and Zhang Y: Melatonin: A well-documented antioxidant with conditional pro-oxidant actions. J Pineal Res 57: 131-146, 2014

115. Saito R, Muneta T, Ozeki N, Nakagawa Y, Udo M, Yanagisawa K, Tsuji K, Tomita M, Koga H and Sekiya I: Strenuous running exacerbates knee cartilage erosion induced by low amount of mono-iodoacetate in rats. BMC Musculoskelet Disord 18: 36, 2017.

116. Gustafsson A and Asman B: Increased release of free oxygen radicals from peripheral neutrophils in adult periodontitis after Fc delta-receptor stimulation. J Clin Periodontol 23: 38-44, 1996

117. Battino M, Bullon P, Wilson $M$ and Newman H: Oxidative injury and inflammatory periodontal diseases: The challenge of anti-oxidants to free radicals and reactive oxygen species. Crit Rev Oral Biol Med 10: 458-476, 1999.

118. Walters J and Lai PC: Should antibiotics be prescribed to treat chronic periodontitis? Dent Clin North Am 59: 919-933, 2015.

119. Srinath R, Acharya AB and Thakur SL: Salivary and gingival crevicular fluid melatonin in periodontal health and disease. J Periodontol 81: 277-283, 2010

120. Gómez-Moreno G, Cutando-Soriano A, Arana C, Galindo P, Bolaños J, Acuña-Castroviejo D and Wang HL: Melatonin expression in periodontal disease. J Periodontal Res 42: 536-540, 2007.

121. Laakso ML, Porkka-Heiskanen T, Alila A, Stenberg D and Johansson G: Correlation between salivary and serum melatonin: Dependence on serum melatonin levels. J Pineal Res 9: $39-50,1990$

122. Madapusi BT and Rao SR: Preliminary evaluation of human gingiva as an extrapineal site of melatonin biosynthesis in states of periodontal health and disease. J Clin Diagnostic Res 12: ZF1-ZF7, 2018

123. Ghallab NA, Hamdy E and Shaker OG: Malondialdehyde, superoxide dismutase and melatonin levels in gingival crevicular fluid of aggressive and chronic periodontitis patients. Aust Dent J 61 : 53-61, 2016.

124. Lodhi K, Saimbi CS, Khan MA, Nath C and Shukla R: Evaluation of melatonin levels in saliva in gingivitis and periodontitis cases: A pilot study. Contemp Clin Dent 7: 519-523, 2016.

125. Cutando A, Galindo P, Gómez-Moreno G, Arana C, Bolaños J, Acuña-Castroviejo D and Wang HL: Relationship between salivary melatonin and severity of periodontal disease. J Periodontol 77: 1533-1538, 2006.

126. Almughrabi OM, Marzouk KM, Hasanato RM and Shafik SS: Melatonin levels in periodontal health and disease. J Periodont Res 48: 315-321, 2013.

127. Tinto M, Sartori M, Pizzi I, Verga A and Longoni S: Melatonin as host modulating agent supporting nonsurgical periodontal therapy in patients affected by untreated severe periodontitis: A preliminary randomized, triple-blind, placebo-controlled study. J Periodont Res 55: 61-67, 2020.

128. Chitsazi M, Faramarzie M, Sadighi M, Shirmohammadi A and Hashemzadeh A: Effects of adjective use of melatonin and vitamin $\mathrm{C}$ in the treatment of chronic periodontitis: A randomized clinical trial. J Dent Res Dent Clin Dent Prospects 11: 236-240, 2017.

129. Bazyar H, Gholinezhad H, Moradi L, Salehi P, Abadi F, Ravanbakhsh M and Zare Javid A: The effects of melatonin supplementation in adjunct with non-surgical periodontal therapy on periodontal status, serum melatonin and inflammatory markers in type 2 diabetes mellitus patients with chronic periodontitis: A double-blind, placebo-controlled trial Inflammopharmacology 27: 67-76, 2019.

130. Sarıtekin E, Üreyen Kaya B, Aşc1 H and Özmen Ö: Anti-inflammatory and antiresorptive functions of melatonin on experimentally induced periapical lesions. Int Endod J 52: 1466-1478, 2019.

131. Renn TY, Huang YK, Feng SW, Wang HW, Lee WF, Lin CT, Burnouf T, Chen LY, Kao PF and Chang HM: Prophylactic supplement with melatonin successfully suppresses the pathogenesis of periodontitis through normalizing RANKL/OPG ratio and depressing the TLR4/MyD88 signaling pathway. J Pineal Res 64: 2018.
132. Botero JE, Yepes FL, Roldán N, Castrillón CA, Hincapie JP Ochoa SP, Ospina CA, Becerra MA, Jaramillo A, Gutierrez SJ and Contreras A: Tooth and periodontal clinical attachment loss are associated with hyperglycemia in patients with diabetes. J Periodontol 83: 1245-1250, 2012.

133. Daniel R, Gokulanathan S, Shanmugasundaram N, Lakshmigandhan $\mathrm{M}$ and Kavin T: Diabetes and periodontal disease. J Pharm Bioallied Sci 4 (Suppl 2): S280-S282, 2012.

134. Grover HS and Luthra S: Molecular mechanisms involved in the bidirectional relationship between diabetes mellitus and periodontal disease. J Indian Soc Periodontol 17: 292-301, 2013

135. Abdolsamadi H, Goodarzi MT, Ahmadi Motemayel F, Jazaeri M,Feradmal J,Zarabadi M, Hoseyni M and Torkzaban P: Reduction of melatonin level in patients with type II diabetes and periodontal diseases. J Dent Res Dent Clin Dent Prospects 8: 160-165, 2014

136. Balci Yuce H, Karatas O, Aydemir Turkal H, Pirim Gorgun E, Ocakli S, Benli I and Cayli S: The effect of melatonin on bone loss, diabetic control, and apoptosis in rats with diabetes with ligature-induced periodontitis. J Periodontol 87: e35-e43, 2016.

137. Cutando A, López-Valverde A, de Diego RG, de Vicente J, Reiter R, Fernández MH and Ferrera MJ: Effect of topical application of melatonin to the gingiva on salivary osteoprotegerin RANKL and melatonin levels in patients with diabetes and periodontal disease. Odontology 102: 290-296, 2014.

138. Montero J, López-Valverde N,Ferrera MJ and López-Valverde A: Changes in crevicular cytokines after application of melatonin in patients with periodontal disease. J Clin Exp Dent 9 e1081-e1087, 2017.

139. Martens L, De Smet S, Yusof MY and Rajasekharan S: Association between overweight/obesity and periodontal disease in children and adolescents: A systematic review and meta-analysis. Eur Arch Paediatr Dent 18: 69-82, 2017.

140. Keller A, Rohde JF, Raymond K and Heitmann BL: Association between periodontal disease and overweight and obesity: A systematic review. J Periodontol 86: 766-776, 2015.

141. Virto L, Cano P, Jiménez-Ortega V, Fernández-Mateos P, González J, Esquifino AI and Sanz M: Obesity and periodontitis: An experimental study to evaluate periodontal and systemic effects of comorbidity. J Periodontol 89: 176-185, 2018.

142. Shimizu I, Yoshida Y and Minamino T: A role for circadian clock in metabolic disease. Hypertens Res 39: 483-491, 2016.

143. Zisapel N: New perspectives on the role of melatonin in human sleep, circadian rhythms and their regulation. $\mathrm{Br}$ J Pharmacol 175: 3190-3199, 2018.

144. Szewczyk-Golec K, Woźniak A and Reiter RJ: Inter-relationships of the chronobiotic, melatonin, with leptin and adiponectin Implications for obesity. J Pineal Res 59: 277-291, 2015.

145. Ríos-Lugo MJ,CanoP,Jiménez-Ortega V,Fernández-Mateos MP, Scacchi PA, Cardinali DP and Esquifino AI: Melatonin effect on plasma adiponectin, leptin, insulin, glucose, triglycerides and cholesterol in normal and high fat-fed rats. J Pineal Res 49 342-348, 2010

146. Favero G, Stacchiotti A, Castrezzati S, Bonomini F, Albanese M Rezzani R and Rodella LF: Melatonin reduces obesity and restores adipokine patterns and metabolism in obese (ob/ob) mice. Nutr Res 35: 891-900, 2015.

147. Virto L, Haugen HJ, Fernández-Mateos P, Cano P, González J, Jiménez-Ortega V, Esquifino AI and Sanz M: Melatonin expression in periodontitis and obesity: An experimental in-vivo investigation. J Periodont Res 53: 825-831, 2018

148. Virto L, Cano P, Jiménez-Ortega V, Fernández-Mateos P, González J, Haugen HJ, Esquifino AI and Sanz M: Melatonin as adjunctive therapy in the treatment of periodontitis associated with obesity. J Clin Periodontol 45: 1336-1346, 2018.

149. Santos RMD, Marani F, Chiba FY, Mattera MSLC, Tsosura TVS, Tessarin GWL, Pereira RF, Belardi BE, Pinheiro BCES and Sumida DH: Melatonin promotes reduction in TNF levels and improves the lipid profile and insulin sensitivity in pinealectomized rats with periodontal disease. Life Sci 213: 32-39, 2018

150. Diomede F, Zingariello M, Cavalcanti MFXB, Merciaro I, Pizzicannella J, De Isla N, Caputi S, Ballerini P and Trubiani O MyD88/ERK/NFkB pathways and pro-inflammatory cytokines release in periodontal ligament stem cells stimulated by Porphyromonas gingivalis. Eur J Histochem 61: 2791, 2017.

151. Nagata M, Iwasaki K, Akazawa K, Komaki M, Yokoyama N, Izumi $\mathrm{Y}$ and Morita I: Conditioned medium from periodontal ligament stem cells enhances periodontal regeneration. Tissue Eng Part A 23: 367-377, 2017. 
152. Bae WJ, Park JS, Kang SK, Kwon IK and Kim EC: Effects of melatonin and its underlying mechanism on ethanol-stimulated senescence and osteoclastic differentiation in human periodontal ligament cells and cementoblasts. Int J Mol Sci 19: 1742, 2018.

153. El-Sharkawy H, Elmeadawy S, Elshinnawi U and Anees M: Is dietary melatonin supplementation a viable adjunctive therapy for chronic periodontitis?-A randomized controlled clinical trial. J Periodont Res 54: 190-197, 2019.

154. Irwin MR, Olmstead R and Carroll JE: Sleep disturbance, sleep duration, and inflammation: A Systematic review and meta-analysis of cohort studies and experimental sleep deprivation. Biol Psychiatry 80: 40-52, 2016.

155. Andersen LP, Gögenur I, Rosenberg J and Reiter RJ: The safety of melatonin in humans. Clin Drug Investig 36: 169-175, 2016

156. Foley HM and Steel AE: Adverse events associated with oral administration of melatonin: A critical systematic review of clinical evidence. Complement Ther Med 42: 65-81, 2019.

157. Frisher M, Gibbons N, Bashford J, Chapman S and Weich S: Melatonin, hypnotics and their association with fracture: A matched cohort study. Age Ageing 45: 801-806, 2016.

158. Sun T, Li J, Xing HL, Tao ZS and Yang M: Melatonin improves the osseointegration of hydroxyapatite-coated titanium implants in senile female rats. Z Gerontol Geriatr 53: 770-777, 2020.
159. Cutando A, Arana C, Gómez-Moreno G, Escames G, López A, Ferrera MJ, Reiter RJ and Acuña-Castroviejo D: Local application of melatonin into alveolar sockets of beagle dogs reduces tooth removal-induced oxidative stress. J Periodontol 78: 576-583, 2007.

160. Swanson CM, Kohrt WM, Buxton OM, Everson CA, Wright KP Jr, Orwoll ES and Shea SA: The importance of the circadian system \& sleep for bone health. Metabolism 84: 28-43, 2018.

161. Wu QY, Wang J, Tong X, Chen J, Wang B, Miao ZN, Li X, Ye JX and Yuan FL: Emerging role of circadian rhythm in bone remodeling. J Mol Med (Berl) 97: 19-24, 2019.

162. Yang N and Meng QJ: Circadian clocks in articular cartilage and bone: A compass in the sea of matrices. J Biol Rhythms 31: 415-427, 2016

163. Tian Y and Li LM: Epidemiological study of sleep disorder in the elderly. Zhonghua Liu Xing Bing Xue Za Zhi 38: 988-992, 2017 (In Chinese).

164. Santoro N: Perimenopause: From research to practice. J Womens Health (Larchmt) 25: 332-339, 2016.

This work is licensed under a Creative Commons Attribution-NonCommercial-NoDerivatives 4.0 International (CC BY-NC-ND 4.0) License. 